\title{
Knockdown of SIRT1 Suppresses Bladder Cancer Cell Proliferation and Migration and Induces Cell Cycle Arrest and Antioxidant Response through FOXO3a-Mediated Pathways
}

\author{
Qingxuan Hu, ${ }^{1}$ Gang Wang, ${ }^{1}$ Jianping Peng, ${ }^{1}$ Guofeng Qian, ${ }^{2}$ Wei Jiang, ${ }^{3,4}$ \\ Conghua Xie, ${ }^{5}$ Yu Xiao, ${ }^{1,4,6}$ and Xinghuan Wang ${ }^{1}$ \\ ${ }^{1}$ Department of Urology, Zhongnan Hospital of Wuhan University, Wuhan, China \\ ${ }^{2}$ Department of Endocrinology, The First Affiliated Hospital of Zhejiang University, Hangzhou, China \\ ${ }^{3}$ Medical Research Institute, Wuhan University, Wuhan, China \\ ${ }^{4}$ Center for Medical Science Research, Zhongnan Hospital of Wuhan University, Wuhan, China \\ ${ }^{5}$ Department of Radiation and Medical Oncology, Zhongnan Hospital of Wuhan University, Wuhan, China \\ ${ }^{6}$ Department of Biological Repositories, Zhongnan Hospital of Wuhan University, Wuhan, China
}

Correspondence should be addressed to Yu Xiao; yu.xiao@whu.edu.cn and Xinghuan Wang; wangxinghuan@whu.edu.cn

Received 4 May 2017; Accepted 7 August 2017; Published 25 September 2017

Academic Editor: Antoni Camins

Copyright (C) 2017 Qingxuan Hu et al. This is an open access article distributed under the Creative Commons Attribution License, which permits unrestricted use, distribution, and reproduction in any medium, provided the original work is properly cited.

\begin{abstract}
Bladder cancer $(\mathrm{BCa})$ is one of the most common tumors, but its underlying mechanism has not been fully clarified. Our transcriptome analysis suggested a close link of Sirtuins, Peroxisome Proliferator-Activated Receptor (PPAR), cell cycle regulation, reactive oxygen species (ROS) metabolism, and Forkhead Box Class O (FOXO) signaling pathway in BCa. SIRT1 is a key member of Sirtuins, playing important roles in aging and energy metabolism, which has been reported to be involved in various metabolic diseases and tumors. We observed that SIRT1 was upregulated in BCa tissues at both mRNA and protein levels. By establishing a SIRT1-knockdown BCa cell model, our results suggested that proliferation and viability were suppressed. Moreover, migration rate was inhibited as well, possibly via reduction of epithelial-mesenchymal transition (EMT). In addition, cell cycle arrest was significantly induced, consisting with strongly decreased proteins involved (CDK2/4/6). Furthermore, ROS production was slightly reduced, accompanied by increasing of antioxidant enzymes and total/acetylated FOXO3a. Consistently with our Path-net analysis, we observed no significant alteration of apoptosis in the SIRT1-knockdown BCa cells. Taken together, our results suggested that SIRT1 deficiency in BCa cells could suppress cell viability by activating antioxidant response and inducing cell cycle arrest possibly via FOXO3a-related pathways.
\end{abstract}

\section{Introduction}

Bladder cancer ( $\mathrm{BCa}$ ), also named urinary bladder cancer, is one of the most common tumors ranking as the 9th leading cause of death worldwide $[1,2]$. A horrible threat as it is to human health, its underlying mechanism, especially its metabolic alterations, has not been fully clarified yet [3-5].

Sirtuins, also known as Sir2-like proteins, are a family of $\mathrm{NAD}^{+}$-dependent deacetylases and ADP-ribosyltransferases [6], playing a vital role in aging [7]. Sir2 was first discovered in yeast via a model of replicative lifespan [8], and later it was shown that addition of an extra copy of the SIR2 gene could extend replicative lifespan by $40 \%$ while deleting SIR 2 shortened lifespan [9].

Mammals contain seven Sirtuins (SIRT1-7), which are categorized by their highly conserved central $\mathrm{NAD}^{+}$-binding and catalytic domain, termed the Sirtuin core domain [10]. And they are involved in many vital processes of cells including oxidative stress response, cell cycle regulation, aging, cell differentiation, energy metabolism, genomic stability, and tumorigenesis $[11,12]$. Among them, Sirt1 deacetylates FOXO family members, $\mathrm{p} 300$, nuclear factor- $\kappa \mathrm{B}(\mathrm{NF}-\kappa \mathrm{B}), \mathrm{p} 53$, and histones $[12,13]$, which regulate cell survival and cellular 
stress response. It also regulates Peroxisome ProliferatorActivated Receptor- $\gamma$, mTOR, and $5^{\prime}$-AMP-dependent kinase (AMPK), which play roles in cellular energy metabolism and autophagy $[11,12]$. SIRT1 is already known related to many metabolic diseases, such as obesity [14-16], diabetes [17-19], and nonalcoholic fatty liver [19-21]. And in respect of cancer, SIRT1 has been reported to be involved in breast cancer [2224], ovarian cancer [25-27], cervical cancer [28-30], gastric cancer [31-33], and prostate cancer [34-36]. But its role in bladder cancer remains largely unknown.

Recent studies of our group based on a transcriptome analysis using human bladder cancer tissues compared with normal bladder tissues [37-39], indicating a close correlation of Sirtuin family, PPAR signaling pathway, cell cycle regulation, ROS metabolism, and FOXO signaling pathway in BCa. Therefore, this study aims to investigate the effect of SIRT1 in $\mathrm{BCa}$ and the underlying mechanism.

\section{Materials and Methods}

2.1. Ethical Statement for Human Bladder Tissue Samples. Human bladder cancer tissue samples $(n=19)$ and human paracancerous tissues $(n=19)$ were all collected from patients suffering bladder cancer in surgery. And all normal bladder tissue samples $(n=4)$ were obtained from donors who died from accident. The samples were either stored in liquid nitrogen for later RNA isolation or fixed in $4 \%$ concentration paraformaldehyde (PFA) for the followed immunofluorescence staining analysis. The Ethics Committee at Zhongnan Hospital of Wuhan University approved the experiments using human bladder tissue samples for RNA and immunofluorescence staining analyses (approval number: 2015029, see Supplementary Material available online at https://doi.org/10.1155/2017/3781904). All methods used for human bladder tissue samples were performed in accordance with the approved guidelines and regulations. Written informed consent was obtained from all subjects.

2.2. Human Bladder Cancer Cells. Human bladder cancer cell line EJ (contaminated by T24 as per "http://iclac.org/databases/ cross-contaminations/") was from the Procell Co. Ltd. (carcinoma, Cat. \#CL-0274) in Wuhan, China. And human bladder cancer cell line T24 (transitional cell carcinoma, Cat. \#SCSP-536) was friendly provided by the Stem Cell Bank, Chinese Academy of Sciences in Shanghai, China. Both T24 and EJ (contaminated by T24 as per "http://iclac.org/databases/ cross-contaminations/") cells were cultured in a humidified atmosphere consisting of $95 \%$ air and $5 \% \mathrm{CO}_{2}$ at $37^{\circ} \mathrm{C}$ in RPMI-1640 medium (Gibco, China) with $10 \%$ fetal bovine serum (FBS) (Gibco, Australia) and 1\% penicillin G sodium/streptomycin sulphate inside.

\subsection{RNA Expression Analyses}

2.3.1. Total RNA Isolation from Bladder Cells and Tissues. We isolated total RNA from cultured bladder cells and collected bladder tissues using Qiagen RNeasy Mini Kit (Cat. \#74101, Qiagen Ltd., Germany), combined with QIAshredder from Qiagen (Cat. \#79654, Qiagen Ltd., Germany), using a centrifuge (Cat. \#5424, Eppendorf Ltd., Germany) according to the manufacturer's protocol. DNase I (RNase-Free DNase Set, Cat. \#79254, Qiagen Ltd., Germany) was used to remove contamination of gDNA from the RNA samples. Concentration of isolated RNA was measured with NanoPhotometer (Cat. \#N60, Implen Ltd., Germany).

2.3.2. Microarray Analysis of $m R N A$ Isolated from Human Bladder Tissues. As previously reported by Cao et al. [37], Wang et al. [38], and Qian et al. [39] from our group, a transcriptome analysis was established by using three human BCa versus three normal bladder tissues. Briefly, biotinylated cDNA were prepared from $250 \mathrm{ng}$ total RNA using the Ambion ${ }^{\circledR}$ WT Expression Kit. Then, $5.5 \mu \mathrm{g}$ cDNA were hybridized on GeneChip Human Transcriptome Array $2.0\left(16 \mathrm{~h}\right.$ at $\left.45^{\circ} \mathrm{C}\right)$ in Hybridization Oven 645. GeneChips were then washed and stained in the Affymetrix Fluidics Station 450 and scanned by Affymetrix ${ }^{\circledR}$ GeneChip Command Console (AGCC), installed in GeneChip ${ }^{\circledR}$ Scanner 3000 (7G). Data were analyzed by Robust Multichip Analysis (RMA). $\mathrm{BCa}$ related genes and pathways were analyzed by using Gene ontology (GO) and Go-map network analysis based on the Gene Cloud of Biotechnology Information software (GCBI System, Shanghai, China) (https://www.gcbi.com.cn) [40]. Thereafter, the gene list was subjected to the Database for Annotation, Visualization and Integrated Discovery (DAVID) [41] for annotation and overrepresentation analysis of the genes involved FOXO signaling pathway (map04068, KEGG pathway image, Kanehisa Laboratories, Japan [42, 43]). The microarray data was uploaded to the Gene Expression Omnibus (GEO) database with accession number: GSE76211. All data are MIAME compliant.

2.3.3. Reverse Transcription and Quantitative Real-Time PCR ( $q R T-P C R)$. Reverse transcription was conducted on an iCycler (Cat. \#CFX Connect, Bio-Rad Ltd., USA) with ReverTra Ace qPCR RT Kit (Toyobo Ltd., China) using $1 \mu \mathrm{g}$ of total RNA mentioned above as template strand. Realtime polymerase chain reactions (qRT-PCR) were performed with iQTM SYBR ${ }^{\circledR}$ Green Supermix (Bio-Rad Ltd., China) in a reaction system of $20 \mu \mathrm{l}$ total volume using $1 \mu \mathrm{g}$ of cDNA. Before qRT-PCR all primers had been tested for optimal annealing temperatures with gradient PCRs. Primer sequences and annealing temperatures used in qRT-PCR are listed in Table 1. Values of GAPDH were used for normalizing amplification. To be more scientific, we used relative gene abundance for further statistical analyses: $\Delta \mathrm{ct}=$ $\mathrm{ct}_{\text {target gene }}-\mathrm{ct}_{\mathrm{GAPDH}}$, for BCa cells $\Delta \Delta \mathrm{ct}=\Delta \mathrm{ct}_{\text {siRNA-treated }}-$ $\Delta \mathrm{ct}_{\text {siRNA-untreated }}$, for bladder tissues $\Delta \Delta \mathrm{ct}=\Delta \mathrm{ct}_{\mathrm{BCa} \text { patients }}-$ $\Delta \mathrm{ct}_{\text {paracancerous tissues }}$, and relative gene abundance $=2^{-\Delta \Delta \mathrm{ct}}$ (ct $=$ threshold cycle).

\subsection{Cell Culture Experiments}

2.4.1. Knockdown of SIRT1 in the BCa Cells. Negative-controlsiRNA and three distinct SIRT1-target specific small interfering RNA (siRNA) were all synthesized by ViewSolid Ltd. in Beijing, China. The sequences of siRNA mentioned are listed in Table 2. These RNA were used to transfect BCa cell lines with LipoJet ${ }^{\mathrm{TM}}$ (SignaGen Ltd., China), according to the 
TABLE 1: List of primers for qRT-PCR.

\begin{tabular}{|c|c|c|c|c|c|}
\hline Gene & Symbol & Forward primer $\left(5^{\prime}-3^{\prime}\right)$ & Reverse primer $\left(5^{\prime}-3^{\prime}\right)$ & $\begin{array}{c}\text { Annealing } \\
\text { temperature } \\
\left({ }^{\circ} \mathrm{C}\right)\end{array}$ & $\begin{array}{c}\text { Length } \\
\text { (bp) }\end{array}$ \\
\hline Sirtuin 1 & SIRT1 & 5'-TAGCCTTGTCAGATAAGGAAGGA-3' & 5'-ACAGCTTCACAGTCAACTTTGT-3' & 58 & 160 \\
\hline $\begin{array}{l}\text { Glyceraldehyde- } \\
\text { 3-phosphate } \\
\text { dehydrogenase }\end{array}$ & $G A P D H$ & $5^{\prime}$-ACAACTTTGGTATCGTGGAAGG-3' & $5^{\prime}$-GCCATCACGCCACAGTTTC- $3^{\prime}$ & 56 & 101 \\
\hline
\end{tabular}

TABLE 2: List of siRNA.

\begin{tabular}{lcccc}
\hline Mark name & Suppressed gene & Sense sequence $\left(5^{\prime}-3^{\prime}\right)$ & Antisense sequence $\left(5^{\prime}-3^{\prime}\right)$ & Supplier \\
\hline NC & Negative control & UUCUCCGAACGUGUCACGUTT & ACGUGACACGUUCGGAGAATT & Viewsolid Biotech, Beijing \\
$s i-1$ & SIRT1 & GGAAAUAUAUCCUGGACAATT & UUGUCCAGGAUAUAUUUCCTT & Viewsolid Biotech, Beijing \\
$s i-2$ & SIRT1 & GCAACUAUACCCAGAACAUTT & AUGUUCUGGGUAUAGUUGCTT & Viewsolid Biotech, Beijing \\
si-3 & SIRT1 & GCUGAUGAACCGCUUGCUATT & UAGCAAGCGGUUCAUCAGCTT & Viewsolid Biotech, Beijing \\
\hline
\end{tabular}

manufacturer's protocol. All transfected cells were cultured for another $48 \mathrm{~h}$ after transfection before being submitted in following tests or harvested for isolation of RNA or protein.

2.4.2. ROS Detection by Staining with DCFH-DA. To measure the ROS level in the $\mathrm{BCa}$ cells, we used the fluorescent probe $2^{\prime}, 7^{\prime}$-dichlorofluorescin diacetate (DCFH-DA, SigmaAldrich Ltd., USA) for staining. $48 \mathrm{~h}$ after transfection, the $\mathrm{BCa}$ cells were harvested and centrifuged; then $1 \mathrm{ml}$ serum-free medium containing $10 \mu \mathrm{M}$ DCFH-DA was added (Sigma-Aldrich Ltd., USA). After another incubation at $37^{\circ} \mathrm{C}$ for $30 \mathrm{~min}$ in dark, the $\mathrm{BCa}$ cells were washed by three times to wash away the extracellular DCFH-DA as fully as possible. Then the cells were submitted to flow cytometry for ROS analysis. For microscope photographing, serum-free medium containing both DCFH-DA and DAPI was added to the $\mathrm{BCa}$ cells slides which were then incubated for $30 \mathrm{~min}$ at the room temperature in the dark and washed three times. Microscope photographs were taken using a fluorescence microscope (Cat. \#IX73, Olympus Ltd., Japan).

2.4.3. Transwell Migration Assay. 24-well plate transwell chamber system with $8.0 \mu \mathrm{m}$ pore size (Corning Ltd., USA) was used for transwell assy. BCa cells cultured for $48 \mathrm{~h}$ after transfection were harvested, centrifuged, and resuspended in serum-free medium and before being diluted to a certain density: $1.33 \times 10^{4}$ cells per $100 \mu \mathrm{l}$ for EJ (contaminated by T24 as per "http://iclac.org/databases/cross-contaminations/") and $2 \times 10^{4}$ cells per $100 \mu \mathrm{l}$ for T24 cells. $300 \mu \mathrm{l}$ of suspension liquid was added to upper chamber insert, while the lower chamber was filled with $600 \mu \mathrm{l}$ of $10 \%$ FBS medium. After incubation for $24 \mathrm{~h}$ at $37^{\circ} \mathrm{C}$, we used cotton swabs to scrub away the cells in the upper insert, and cells that migrated to the lower side were fixed by $4 \%$ PFA and stained by $0.1 \%$ crystal violet. After washing away crystal violet and air drying, the chambers were placed under an inverted phase contrast microscope (Cat. \#DMI 1, Leica Ltd., Germany) to take pictures and count the migrated cells.
2.4.4. MTT Assay. $48 \mathrm{~h}$ after transfection, the BCa cells were seeded in 96-well plates (3,000 cells per $200 \mu \mathrm{l}$ medium) to be cultured for another 1-5 days. Every $24 \mathrm{~h}$ one plate was measured during the 5 days. When measuring, $20 \mu \mathrm{l} \mathrm{MTT}$ $(5 \mathrm{mg} / \mathrm{ml})$ was added to each well before measured plate was incubated for another $4 \mathrm{~h}$ at $37^{\circ} \mathrm{C}$, and then the medium was removed, formazan precipitate was dissolved in $150 \mu \mathrm{l}$ DMSO, and absorbance was measured at $490 \mathrm{~nm}$ with a microplate reader (Cat. \#SpectraMax M2, Molecular Devices Ltd., USA).

2.4.5. Clonogenic Forming Experiment. After transfection for $48 \mathrm{~h}$, distinct BCa cells were seeded in 6-well plates $(800$ cells per well) and grew into colonies for approximately 14 days. Then colonies were fixed by $4 \%$ PFA for $30 \mathrm{~min}$, stained with crystal violet for $30 \mathrm{~min}$, washed with PBS, naturally dried, counted, and photographed.

2.4.6. Flow Cytometry Analysis for Alterations of Cell Cycle and Apoptosis. For cell cycle analysis, the BCa cells were transfected by siRNA for $48 \mathrm{~h}$ and then harvested and centrifuged. Thereafter, the cells were washed by PBS for three times and resuspended with 1x DNA staining solution containing propidium iodide and permeabilization solution (Multisciences Ltd., China) in the dark. After another incubation $\left(37^{\circ} \mathrm{C}\right.$ for $\left.30 \mathrm{~min}\right)$, cells were analyzed by flow cytometer (Cat. \#FC500, Beckman Ltd., USA). Apoptosis analysis was assessed with the FITC Annexin V Apoptosis Detection Kit I (BD biosciences Ltd., USA), according to the manufacturer's instructions and analyzed by the flow cytometry analysis.

2.4.7. TUNEL Assay. After $48 \mathrm{~h}$ transfection, coverslips with BCa cells were fixed by $4 \%$ PFA for $30 \mathrm{~min}$ at room temperature and washed three times by ice-cold PBS, continuously incubated with $0.1 \%$ Triton X-100 for $2 \mathrm{~min}$, and washed by PBS for three times. Then, apoptotic cells were measured by the TdT-mediated dUTP-biotin nick end labeling test (TUNEL, Roche Applied Science Ltd., Germany), according to the manufacturer's instructions. Nuclei were stained by 
TABLE 3: List of primary antibodies.

\begin{tabular}{|c|c|c|c|c|}
\hline Antigens & $\begin{array}{l}\text { Species antibodies } \\
\text { raised in }\end{array}$ & Dilution (IF) & Dilution (WB) & Supplier \\
\hline $\begin{array}{l}\text { Acetyllysine, acetylated KLH } \\
\text { conjugates }\end{array}$ & Rabbit, polyclonal & - & $1: 500$ & Abcam, UK, Cat. \#ab80178 \\
\hline Catalase, human & Rabbit, monoclonal & - & $1: 2,000$ & Abcam, UK, Cat. \#ab76024 \\
\hline CDK2, human & Rabbit, monoclonal & - & $1: 2,000$ & Abcam, UK, Cat. \#ab32147 \\
\hline CDK4, human & Rabbit, monoclonal & - & $1: 2,000$ & Abcam, UK, Cat. \#ab124821 \\
\hline CDK6, human & Rabbit, monoclonal & - & $1: 1,000$ & Abcam, Cat. \#ab124821 \\
\hline Cleaved caspase- 3 , human & Rabbit, monoclonal & $1: 200$ & - & Cell Signaling Technology, USA, Cat. \#9664 \\
\hline Cleaved caspase-7, human & Rabbit, monoclonal & $1: 200$ & - & Cell Signaling Technology, USA, Cat. \#8438 \\
\hline Cleaved caspase- 9 , human & Rabbit, monoclonal & $1: 200$ & - & Cell Signaling Technology, USA, Cat. \#7237 \\
\hline FOXO3a, human & Rabbit, monoclonal & - & $1: 1,000$ & Abcam, UK, Cat. \#ab53287 \\
\hline Ki-67, human & Rabbit, monoclonal & $1: 200$ & - & Novus Biologicals, USA, Cat. \#NBP2-19012 \\
\hline OCT-4, human & Mouse, monoclonal & $1: 200$ & - & Novus Biologicals, USA, Cat. \#NB110-90606 \\
\hline p53 (acetyl K370), human & Rabbit, monoclonal & - & $1: 1,000$ & Abcam, UK, Cat. \#ab183544 \\
\hline $\operatorname{PPAR} \gamma$, human & Rabbit, monoclonal & - & $1: 1,000$ & Abcam, UK, Cat. \#ab45036 \\
\hline SIRT1, human & Rabbit, monoclonal & $1: 400$ & $1: 1,000$ & Cell Signaling Technology, USA, Cat. \#9475 \\
\hline SOD2, human & Rabbit, monoclonal & - & $1: 1,000$ & Abcam, UK, Cat. \#ab68155 \\
\hline$\beta$-Actin, human & Mouse, monoclonal & - & $1: 2,000$ & $\begin{array}{c}\text { Santa Cruz Biotechnology Inc., Dallas, TX., USA, } \\
\text { Cat. \#sc-47778 }\end{array}$ \\
\hline E-Cadherin, human & Rabbit, monoclonal & $1: 200$ & - & Cell Signaling Technology, USA, Cat. \#3195 \\
\hline $\mathrm{N}$-Cadherin, human & Rabbit, monoclonal & $1: 200$ & - & Cell Signaling Technology, USA, Cat. \#13116 \\
\hline
\end{tabular}

TABLE 4: List of secondary antibodies and counterstaining of nuclei.

\begin{tabular}{|c|c|c|c|c|}
\hline Secondary detection system used & Host & Method & Dilution & Supplier \\
\hline Anti-mouse-IgG (H + L)-HRP & Goat & WB & $1: 10,000$ & Sungene Biotech, China, Cat. \#LK2003 \\
\hline Anti-rabbit-IgG $(\mathrm{H}+\mathrm{L})-\mathrm{HRP}$ & Goat & WB & $1: 10,000$ & Sungene Biotech, China, Cat. \#LK2001 \\
\hline $\begin{array}{l}\text { Anti-rabbit IgG }(\mathrm{H}+\mathrm{L}), \mathrm{F}\left(\mathrm{ab}^{\prime}\right) 2 \\
\text { fragment (Alexa Fluor }{ }^{\circledR} 488 \text { Conjugate) }\end{array}$ & Goat & IF & $1: 50$ & Cell Signaling Technology, USA, Cat. \#4412 \\
\hline $\begin{array}{l}\text { Anti-mouse IgG }(\mathrm{H}+\mathrm{L}), \mathrm{F}\left(\mathrm{ab}^{\prime}\right) 2 \\
\text { fragment (Alexa Fluor }{ }^{\circledR} 555 \text { Conjugate) }\end{array}$ & Goat & IF & $1: 50$ & Cell Signaling Technology, USA, Cat. \#4408 \\
\hline Anti-goat IgG-FITC & Rabbit & IF & $1: 100$ & Boster Biological Technology, China, Cat. \#BA1110 \\
\hline Anti-goat IgG-Cy3 & Rabbit & IF & $1: 100$ & Boster Biological Technology, China, Cat. \#BA1034 \\
\hline $\begin{array}{l}\text { Hoechst } 33342 \text { nucleic acid staining } \\
\text { (DAPI) }\end{array}$ & - & IF & $1: 750$ & Molecular Probes/Invitrogen, USA, Cat. \#A11007 \\
\hline
\end{tabular}

$1 \mu \mathrm{M}$ DAPI for $20 \mathrm{~min}$ at room temperature. Images were taken using the fluorescence microscope.

\subsection{Protein Analyses}

2.5.1. Isolation of Total Protein from BCa Cells. The RIPA buffer with protease inhibitor and phosphatase inhibitor (Sigma-Aldrich Ltd., USA) was used to lyse and sonicate BCa cells on ice for $30 \mathrm{~min}$ and then centrifuged at $12,000 \mathrm{~g}$ for $15 \mathrm{~min}$ to collect supernatant. The density of protein was measured by the Bradford protein assay (Bio-Rad Ltd., Germany).

2.5.2. Western Blot and Immunoprecipitation Analysis. For the following Western blot analysis, total protein was separated by electrophoresis in 10-12.5\% SDS-PAGE and then transferred to the PVDF membrane (Millipore Ltd., USA). Membranes were then blocked in 5\% fat-free milk and continuously incubated with primary antibodies (Table 3 ) at $4^{\circ} \mathrm{C}$ for overnight and secondary antibodies (Table 4 ) for $2 \mathrm{~h}$ at room temperature. Bands were visualized using an enhanced chemiluminescence (ECL) kit (Bio-Rad Ltd., USA) and detected by ChemiDoc XRS ${ }^{+}$Imaging System (Bio-Rad Ltd., USA).

The immunoprecipitation analysis for acetylation level of FOXO3a was done according to Frazzi et al. [44]. Briefly, total cell lysates were prepared with lysis buffer and cleared by centrifugation. Then, $200 \mu \mathrm{l}$ of cell lysates was incubated with anti-FOXO3a antibody and rotated at $4^{\circ} \mathrm{C}$ for overnight. The samples were continually incubated with protein $\mathrm{A}$ agarose for 2 hours. After washing the immunoprecipitated 
produce was eluted, loaded on $10 \%$ polyacrylamide gels, and performed by SDS-PAGE, as well as detected by the ECL kit. The intensity of bands was measured with the ancillary software of ChemiDoc XRS ${ }^{+}$Imaging System (Bio-Rad Ltd., USA), named Image Lab (version 5.1, build 8). To compare the acetylation level of FOXO3a, we calculated the relative acetylation rate of FOXO3a: the relative acetylation rate = intensity of anti-acetyl Lysine/intensity of total FOXO3a band. The intensity of total FOXO3a was used as a loading control and its rate of NC group was normalized to 1.

\subsubsection{Immunofluorescence Staining for Human Bladder Tissue} Samples. The bladder tissue samples were fixed by $4 \%$ PFA containing $2 \%$ sucrose in PBS at $4^{\circ} \mathrm{C}$ for overnight and embedded into paraffin (Paraplast, Sigma-Aldrich Ltd., USA) using a tissue processor (Cat. \#STP 120, Thermo Fisher Scientific Ltd., UK). Paraffin sections $(4 \mu \mathrm{m})$ were cut with a rotation microtome (Cat. \#HM325, Thermo Fisher Scientific Ltd., Germany). The sections were serially incubated with primary antibody and Cy3-labeled or FITC- labeled secondary antibody in humidified atmosphere (Tables 3 and 4). Nuclei were labeled with DAPI $(2 \mu \mathrm{g} / \mathrm{ml})$. Immunofluorescence staining images for paraffin sections were analyzed by the fluorescence microscope.

2.5.4. Immunofluorescence Analysis for BCa Cells. Coverslips with BCa cells were washed three times with ice-cold PBS and fixed by $4 \%$ PFA for $30 \mathrm{~min}$. Cells were then treated with $0.1 \%$ Triton X-100 solution and blocked using normal goat serum for $30 \mathrm{~min}$ at room temperature. Afterwards, the cells were incubated with the indicated primary antibody (Table 3 ) at the proper dilution for $2 \mathrm{~h}$ at room temperature, washed with PBS for three times, and incubated with Cy3-labeled or FITC labeled secondary antibody (Table 4) for $1 \mathrm{~h}$. Nuclei were stained by $1 \mathrm{mM}$ TOTO-3 iodide for $10 \mathrm{~min}$ at room temperature. Immunofluorescence staining was analyzed by the fluorescence microscope.

2.6. Statistical Analyses. All data described as mean $\pm \mathrm{SD}$ form is from three or more independent experiments. Twotailed Student's paired and unpaired $t$-tests were used to evaluate the statistical significance of the data. All of the statistical analyses were performed with SPSS 16.0. Statistical significance was set at probability values of $p<0.05$.

\section{Results}

3.1. Upregulation of SIRT1 in BCa Tissues Compared with Paracancerous Tissues and Normal Bladder Tissues. qRTPCR analysis was performed to evaluate the expression of SIRT1 gene, indicating significant upregulation in the $\mathrm{BCa}$ tissues compared with the paired paracancerous tissues $(n=$ $16, p<0.05$, Figure 1(a)). Double immunofluorescence staining showed that, in the BCa tissues, SIRT1 protein was strongly increased in the OCT4-positive cells (Figure 1(b)), which has been suggested to be a potential biomarker for $\mathrm{BCa}$ [37], whereas the corresponding paracancerous bladder tissues and the normal bladder tissues exhibited slight staining of both OCT4 and SIRT1 protein (Figure 1(b)), consisting with the qRT-PCR result. Overrepresentation analysis using microarray raw data [37-39] and DAVID database revealed that, in the BCa tissues, increased SIRT1 expression could affect oxidative stress resistance, cell cycle regulation, and energy metabolism, via FOXO signaling pathway (Figure 1(c)).

3.2. SIRT1 Deficiency Affected ROS Level in BCa Cells. A cell model of SIRT1 deficiency was established by siRNAtransfection in the $\mathrm{BCa}$ cells. The $\mathrm{BCa}$ cells were transfected by three distinct SIRT1-target-specific-siRNA (si-1, si2 , and si-3) and negative-control-siRNA (NC) (sequences are listed in Table 1). Efficiency of knockdown was validated by qRT-PCR $48 \mathrm{~h}$ after transfection (Figure 2(a)). Considering qRT-PCR result in both cell lines overall, we used si-2 to perform the following experiments. Knockdown rate was $80.4 \%$ in EJ (contaminated by T24 as per "http://iclac.org/databases/cross-contaminations/") and $86.8 \%$ in T24 cells. We noticed a significant downregulation of SIRT1 protein abundance was observed by Western blot analysis (Figure 2(b)) and immunofluorescence staining (Figure 2(c)), indicating transfection by the SIRT1-target-specificsiRNA si-2 could reduce SIRT1 at both transcriptional and translational levels in both BCa cell lines.

SIRT1 is reported to play a role in the metabolism of the reactive oxygen species (ROS) in cells [36-38], but how SIRT1 affected ROS metabolism in bladder cancer cells has not been reported yet. Therefore, we used DCFH-DA to stain the siSIRT1 group and NC group before flow cytometry analysis. The results suggested that BCa cells lacking SIRT1 exhibited a reduced level of ROS production than the NC group (Figure 2(d)). Consistently, ROS staining using fluorescence staining showed a similar result (Figure 2(e)), accompanied by increasing of distinct antioxidant enzymes (Catalase and SOD2, Figure 2(f)). Moreover, our results suggested that the central transcription receptors (FOXO3a, PPAR $\gamma$, and acetylated p53) were strongly upregulated in the si-SIRT1 group (Figure $2(\mathrm{f})$ ).

3.3. Acetylation Level of FOXO3a Was Induced in the BCa Cells with Downregulated SIRT1. Since no effective antibody for acetylated FOXO3a is available, we used immunoprecipitation experiment to test the acetylation level of FOXO3a (Figure 2(g)). By using the intensity of total FOXO3a as a loading control, the relative acetylation rate of FOXO3a was statistically analyzed (Figure $2(\mathrm{~h})$ ). The results suggested that the acetylation level of FOXO3a in the downregulated SIRT1 group was significantly increased.

\subsection{Knockdown of SIRT1 Inhibited Proliferation and Viability} in BCa Cells and Triggered Cell Cycle Arrest at G0/G1 Phase. Clonogenic survival assay revealed that the ability of clone formation was decreased in the si-SIRT1 cells by $36.5 \%$, comparing with the NC group (Figures 3(a) and 3(b)). Moreover, MTT assay revealed that the si-SIRT1-treated BCa cells grew significantly slower than the negative-control-siRNA-treated group (Figure $3(\mathrm{c})$ ), consisting with strongly reduced Ki-67 positive cells in the si-SIRT1 group (Figure 3(d)), which could be used as an indicator for proliferation [45]. 


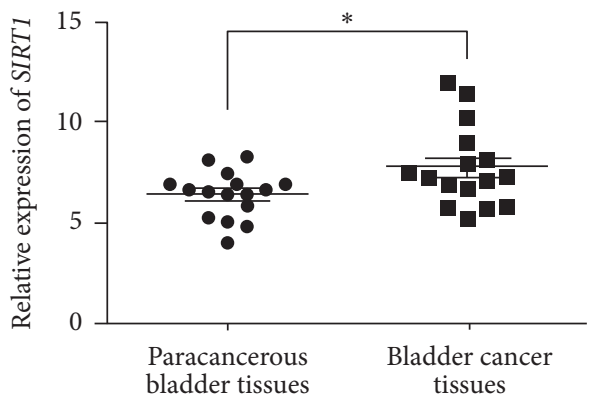

(a)

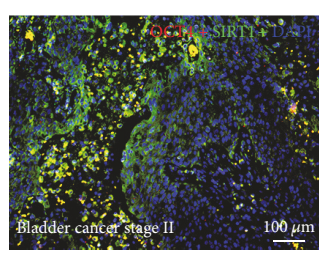

(A)

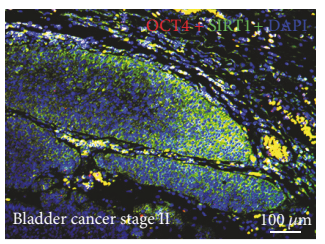

(D)

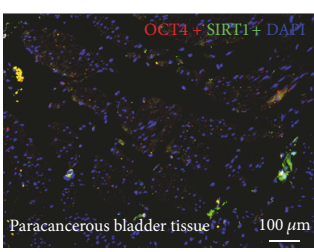

(B)

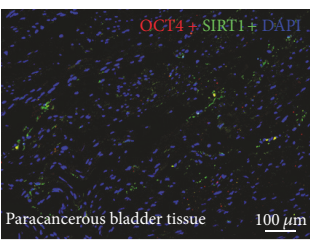

(E)

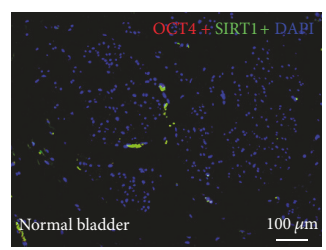

(C)

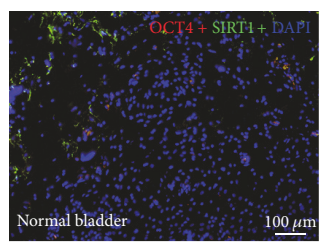

(F)

(b)

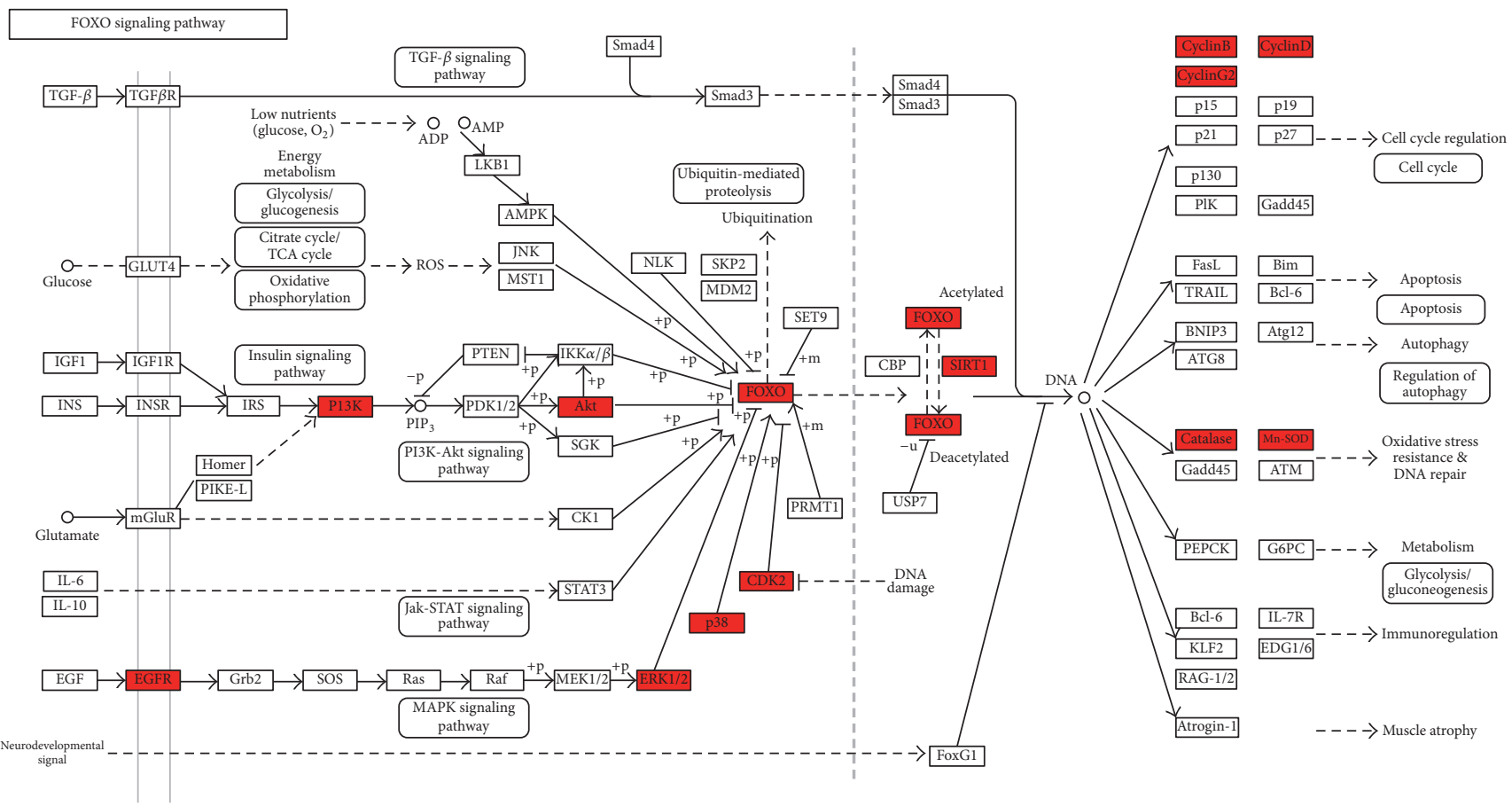

(c)

FIGURE 1: SIRT1 is strongly upregulated in the BCa tissues compared with both paracancerous tissues and normal bladder tissues. (a) qRT-PCR analysis exhibited that the gene expression of SIRT1 in bladder cancer tissues was significantly higher than the matched paracancerous tissues. The value of GAPDH was used as an internal control. ${ }^{*} p<0.05$. (b) Representative result of double immunofluorescence staining of SIRT1 (green) in bladder cancer tissues (A, D), paracancerous tissues (B, E), and normal bladder tissues (C, F). The OCT4 (red) was stained as a marker of BCa cells. Nuclei (blue) were stained by DAPI. The scale bar for (b) is $100 \mu \mathrm{m}$. (c) Overrepresentation analysis using microarray raw data and DAVID database suggested that the FOXO signaling pathway and related genes were affected (red) in the BCa tissues (FOXO signaling pathway, map04068, KEGG pathway image, copyright permission obtained from KEGG).

Flow cytometry analysis of cell cycle indicated that there was higher percentage of cells at G0/G1 phase in si-SIRT1 group than in $\mathrm{NC}$ group while it was on the contrary at $\mathrm{S}$ phase (Figures $3(\mathrm{e})$ and $3(\mathrm{~g})$ ), which suggested that SIRT1 deficiency induced cell cycle arrest at G0/G1 phase. In addition, Western blot result suggested proteins involved in regulating G0/G1 phase (CDK2/4/6) were considerably decreased in si-SIRT1 group (Figure 3(f)).
3.5. Downregulation of SIRT1 Inhibited Migration in BCa Cells. Cell migration was measured using transwell migration assay, and migration rate was significantly lower in si-SIRT1 group than the NC group both in EJ (contaminated by T24 as per "http://iclac.org/databases/cross-contaminations/") and in T24 (Figures 4(a) and 4(b)). Affected by the transfection of SIRT1-target-specific-siRNA, the migration rate declined by $67.5 \%(p<0.05)$ in EJ (contaminated by T24 as per 


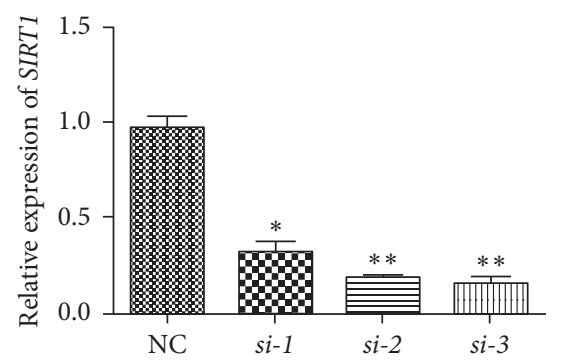

(A)

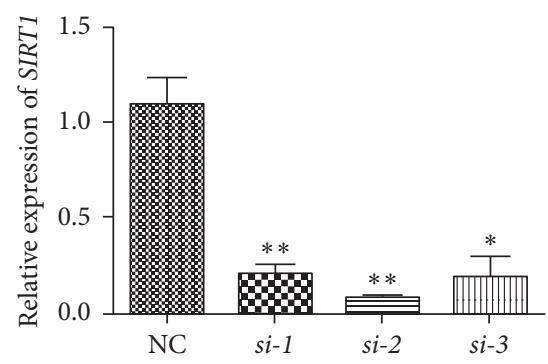

(B)

(a)

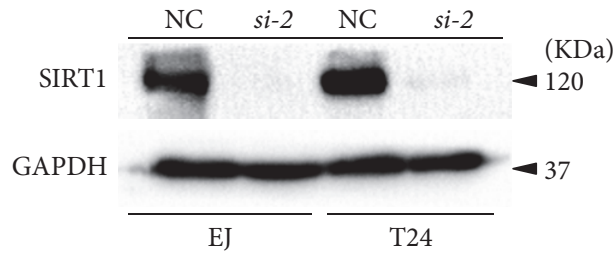

(b)

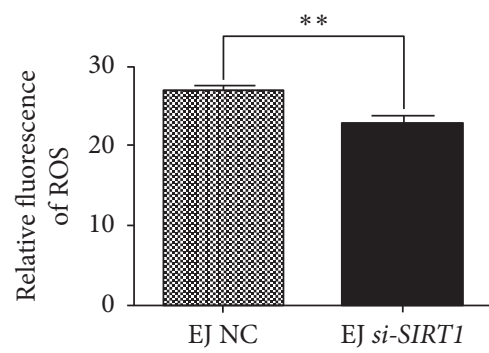

(A)

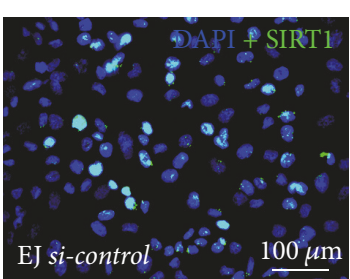

(A)

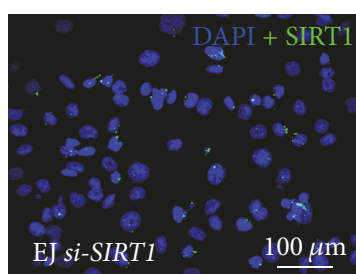

(B)

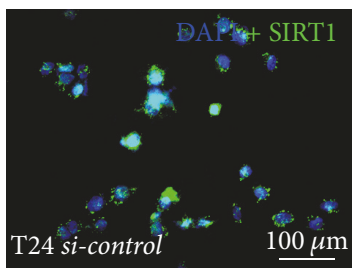

(C)

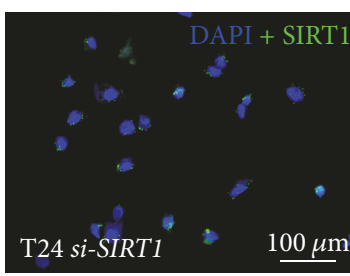

(D)

(c)

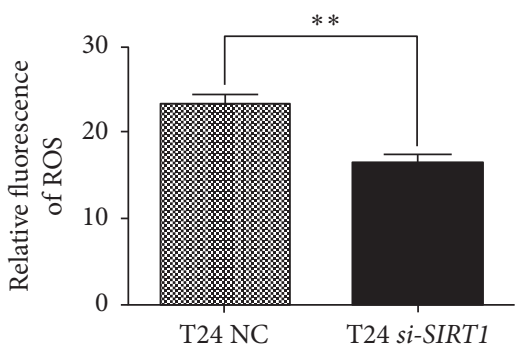

(B)

(d)

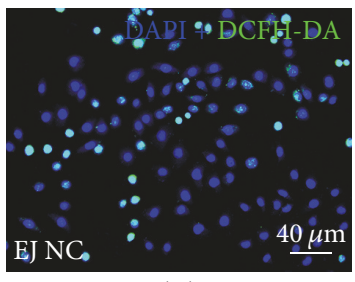

(A)

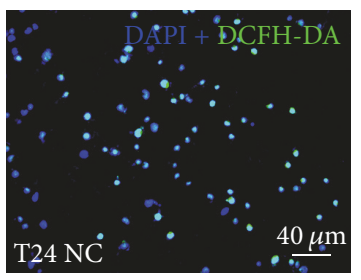

(C)

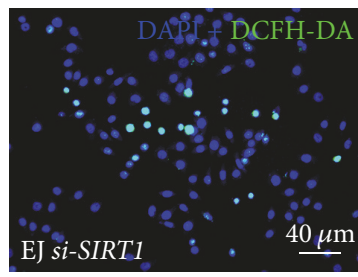

(B)

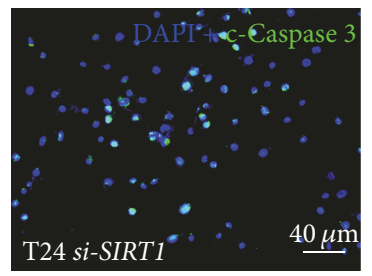

(D)

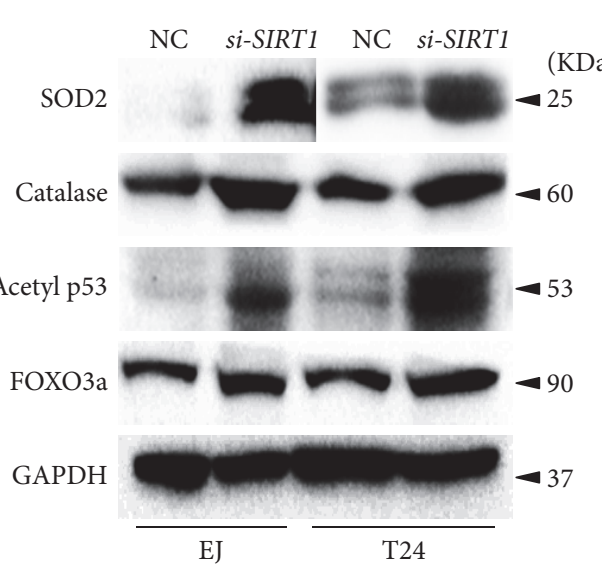

(f)

Figure 2: Continued. 


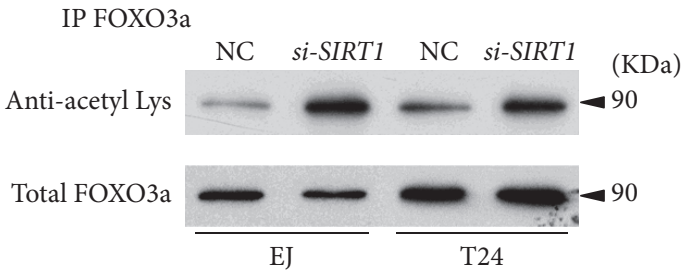

(g)

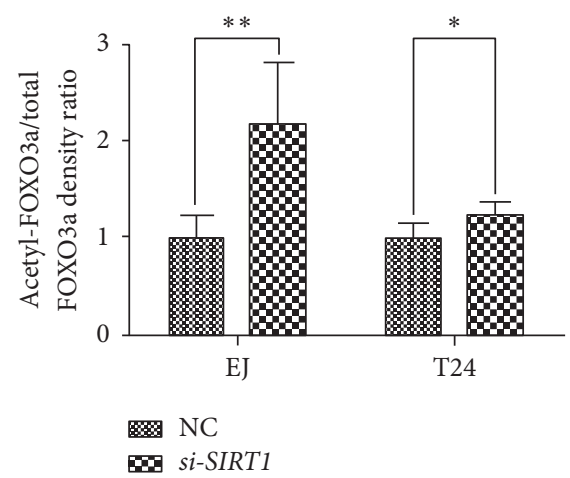

(h)

FIGURE 2: Knockdown of SIRT1 in BCa cells induced alteration of ROS and related proteins. (a) qRT-PCR was used to testify the KD efficiency by using different siRNA to knockdown SIRT1 in T24 (A) and EJ (B) (contaminated by T24 as per "http://iclac.org/databases/cross-contaminations/") bladder cancer cells. All shown values were mean \pm SD of three measurements and repeated three or more times, ${ }^{*} p<0.05$ and ${ }^{* *} p<0.01$. (b) Western blot bands of SIRT1 and GAPDH in EJ (contaminated by T24 as per "http://iclac.org/databases/cross-contaminations/") and T24, SIRT1 deficiency BCa cells compared with NC BCa cells. The GAPDH abundance was used as an internal control. (c) Representative immunofluorescence staining of SIRT1 (green) in the BCa cells after SIRT1target-specific-siRNA treatment (KD) (B, D), compared with control-siRNA treatment (NC) (A, C). Nuclei (blue) were stained by DAPI. The scale bar for (c) is $100 \mu \mathrm{m}$. (d) Statistical analysis of relative fluorescence of ROS in the NC and si-SIRT1 transfected EJ (A) (contaminated by T24 as per "http://iclac.org/databases/cross-contaminations/") and T24 (B) cells. ${ }^{* *} p<0.01$. (e) Representative DCFH-DA staining of ROS (green) in the BCa cells with si-SIRT1 treatment (B, D) versus control-siRNA treatment (NC) (A, C). Nuclei (blue) were stained by DAPI. The scale bar for (e) is $40 \mu \mathrm{m}$. (f) Western blot analyses of antioxidant enzymes (SOD2, Catalase) acetylated p53 and total FOXO3a in the NC and si-SIRT1 transfected EJ (contaminated by T24 as per "http://iclac.org/databases/cross-contaminations/") and T24 cells (cell types, siRNA treatment, and protein masses were indicated). The GAPDH was used as a loading control. 10-30 $\mu \mathrm{g}$ of total protein were loaded per lane. (g) Immunoprecipitation analysis of antiacetylated Lysine and total FOXO3a in the NC and si-SIRT1 transfected EJ (contaminated by T24 as per "http://iclac.org/databases/cross-contaminations/") and T24 cells. (h) Statistical analysis of relative acetylation rate of FOXO3a by measurement of the band intensity of three independent Western blot experiments, calculated as relative acetylation rate of FOXO3a $=$ intensity of antiacetylated Lysine/intensity of total FOXO3a. The intensity of total FOXO3a was used as control and its rate of NC group was normalized to $1 .{ }^{*} p<0.05 ;{ }^{* *} p<0.01$.

"http://iclac.org/databases/cross-contaminations/") and 48.5\% in T24 $(p<0.01)$. Furthermore, the protein abundances of E-cadherin and N-cadherin, involved in the epithelialmesenchymal transition (EMT) process, were affected by reduction of SIRT1. Our results showed that the E-cadherin level was obviously higher and the $\mathrm{N}$-cadherin was lower in si-SIRT1 group (Figures $4(\mathrm{c})$ and $4(\mathrm{~d})$ ), suggesting that activity of EMT process was reduced by SIRT1 deficiency.

3.6. Lower Level of SIRT1 Expression Had No Significant Effect on Apoptosis in $\mathrm{BCa}$ Cells. We also performed flow cytometry analysis to investigate apoptosis alternation of SIRT1 deficiency. But after multiple repeated tests, the result yet suggested no significant alteration between si-SIRT1 group and NC group (Figure 5(a)). To make further affirmation, we conducted TUNEL assay, and the result suggested no significant alteration either (Figure 5(b)). Consistently, in immunofluorescence staining, no significant (n.s.) alteration of the apoptosis related proteins cleaved-Caspases 3, 7, and 9 in si-SIRT1 group was observed (Figures $5(\mathrm{~d})-5(\mathrm{f})$ ).

\section{Discussion}

In our previous studies [37-39] based on comprehensive transcriptome analysis using human bladder cancer tissues compared with normal bladder tissues, we found that SIRT1 played a vital role in tumorigenesis and further development of human bladder cancer (BCa). SIRT1 is a crucial gene in process of aging [9], energy metabolism, and autophagy [11], but its role in BCA remains largely unknown. Our further investigation in human bladder tissue samples at a larger scale revealed that SIRT1 possessed an overexpression in human bladder cancer tissues than in paracancerous tissues or normal bladder tissues, at both transcriptional and protein levels (Figure 1). Therefore, overrepresentation analysis and DAVID database (Figure 1) revealed that SIRT1 could interfere FOXO signaling pathway to affect oxidative stress resistance, cell cycle regulation, and energy metabolism in the BCa tissues.

FOXO proteins constitute a family of transcription factors that play important role in regulating the expression of genes involved in cell growth, proliferation, differentiation, and longevity. FOXO3a is a key submember in the FOXO family involved in $\mathrm{AKT} / \mathrm{FOXO} 3 \mathrm{a} / \beta$-catenin pathway [39], regulating cell cycle, oxidative stress response, and apoptosis [46-48]. In the downregulated SIRT1 BCa cells, total and acetylated FOXO3a were strongly induced (Figure 2), suggesting the correlated cell cycle regulation and antioxidant response were affected. These results were similar as reported by Frazzi et al. [44], which indicated that by using an inhibitor of SIRT1 (resveratrol) the acetylation level of FOXO3a was strongly upregulated in the Hodgkin lymphoma cells. 


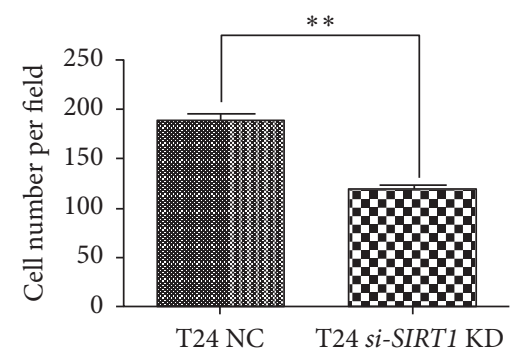

T24 NC

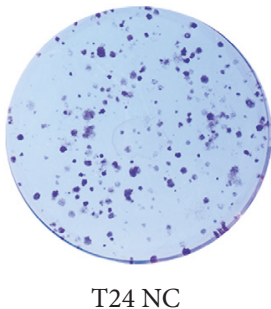

(A)

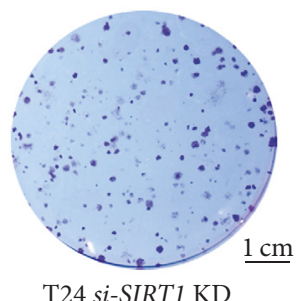

(B)

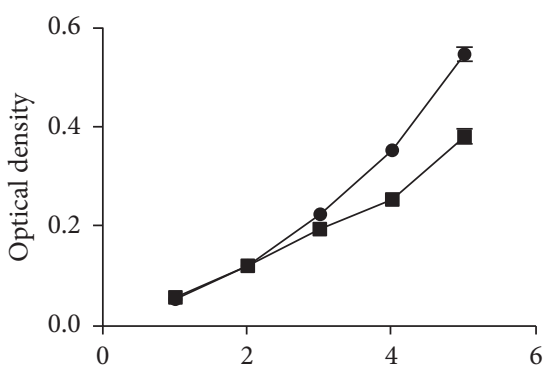

(Days)

$\mathrm{T} 24 \mathrm{NC}$

T24 si-SIRT1 KD

(b)

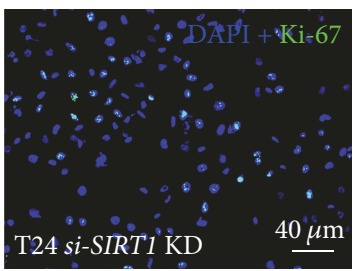

(B)

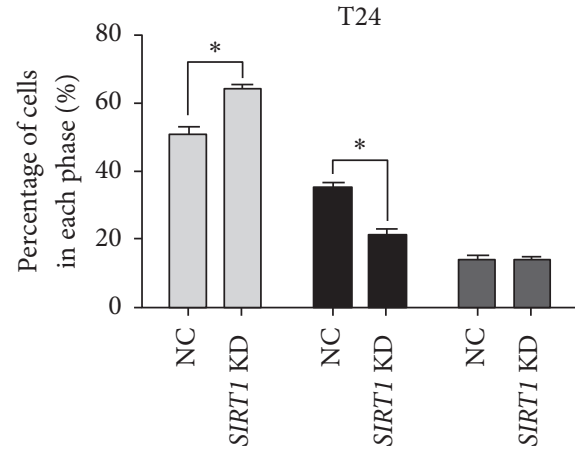

$\square$ G0-G1
S
$\square$ G2-M

(d)

(e)

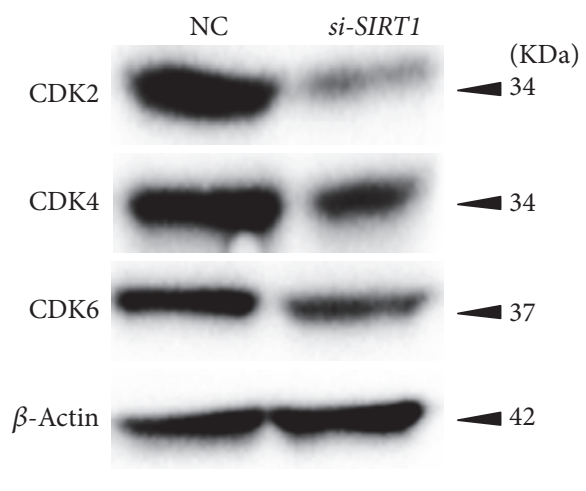

(f)

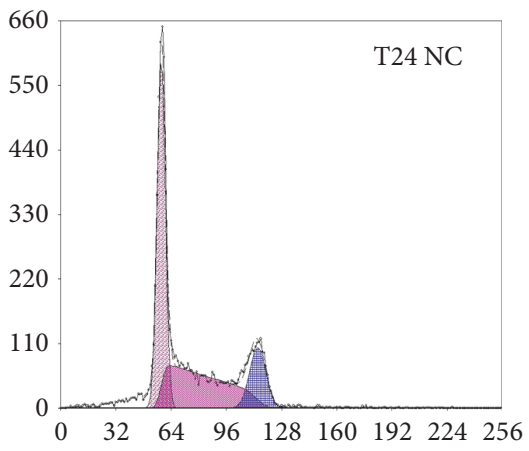

(A)

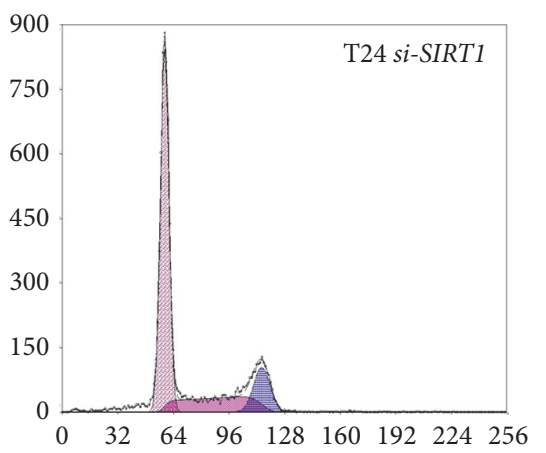

(B)

(g)

FIGURE 3: Downregulation of SIRT1 repressed BCa cell proliferation and induced cell cycle arrest. (a) Clone number in each well was counted and statistically analyzed in the clonogenic survival assay. ${ }^{* *} p<0.01$. (b) Clonogenic survival assay revealed cell survival of BCa cells after treatment of SIRT1-target-specific-siRNA (SIRT1 KD) and control-siRNA (NC), cultured in 6-well plates for 14 days. (c) MTT assay was used to measure the viability of BCa cells treated by SIRT1-target-specific-siRNA (SIRT1 KD, line linking squares) and negative-control-siRNA (NC, line linking circles). All shown values were mean \pm SD of three measurements and repeated three times with similar results, ${ }^{*} p<0.05$. (d) Cell proliferation of BCa cells treated by SIRT1-target-specific-siRNA (B) and negative-control-siRNA (A) was assayed by Ki-67 staining (green). Nuclei (blue) were stained by DAPI. (e) Statistical analysis of percentages (\%) of BCa cell populations at different stages of cell cycles. All shown values were mean $\pm \mathrm{SD}$ of three measurements and repeated three times with similar results. ${ }^{*} p<0.05$. (f) Western blot analysis of proteins involved in G0-G1 cell cycle regulation (CDK2, CDK4, and CDK6) in the BCa cells. $\beta$-Actin abundance was used as a control. (g) Flow cytometry analysis result for BCa cells treated with negative-control-siRNA (A) and SIRT1-target-specific-siRNA (B) for $48 \mathrm{~h}$. The scale bar for (b) is $1 \mathrm{~cm}$ and for (d) is $40 \mu \mathrm{m}$. 


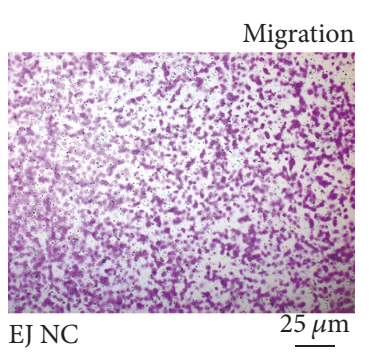

(A)

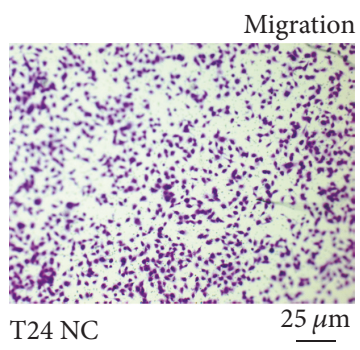

(C)

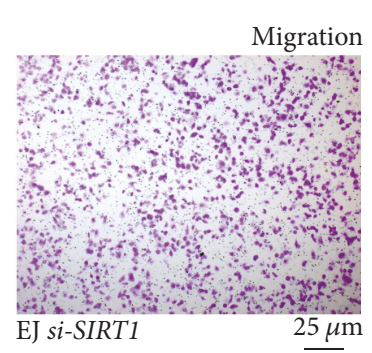

(B)

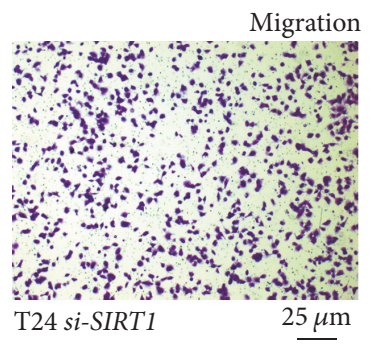

(D)

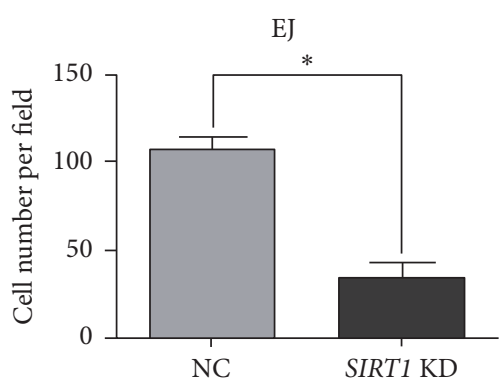

(A)

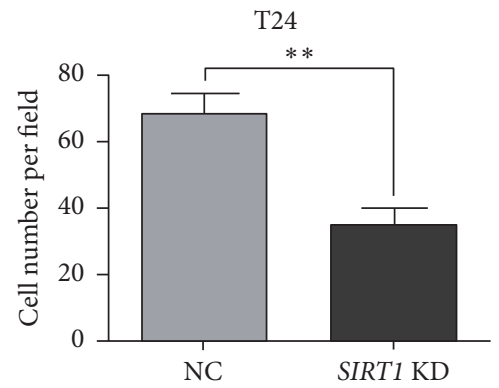

(B)

(a)

(b)

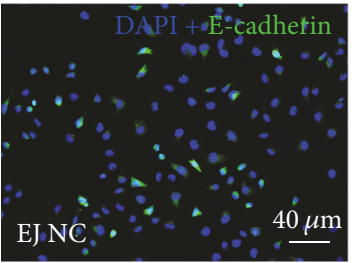

(A)

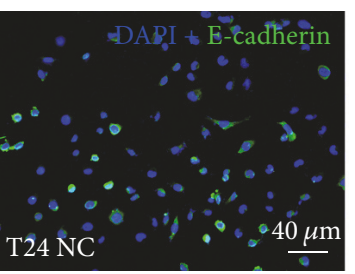

(C)

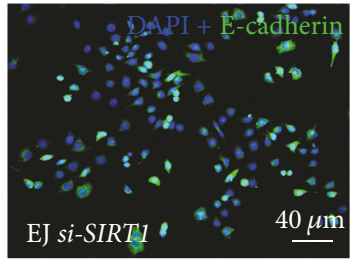

(B)

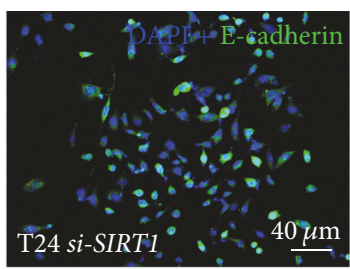

(D)

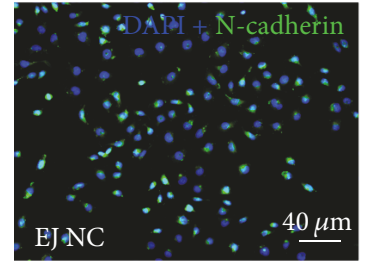

(A)

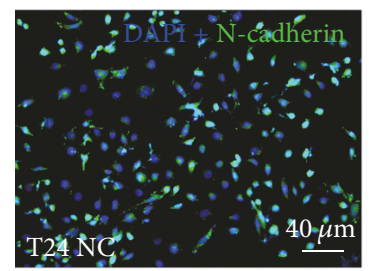

(C)

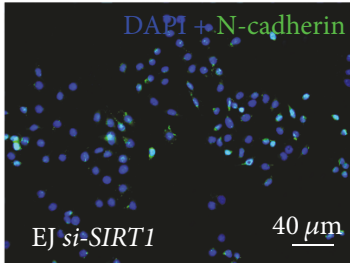

(B)

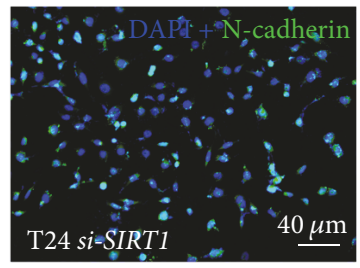

(D)

(c)

(d)

FIGURE 4: SIRT1 deficiency inhibited migration of BCa cells. (a) Transwell migration assay was conducted to measure the migration ability of NC group cells (A, C) and si-SIRT1 group cells (B, D) in EJ (contaminated by T24 as per "http://iclac.org/databases/cross-contaminations/") and T24 for $48 \mathrm{~h}$ after transfection. (b) BCa cells in transwell migration assay were counted on microscope and statistically analyzed. All shown values were mean $\pm \mathrm{SD}$ of three measurements and repeated three times with similar results. ${ }^{*} p<0.05 ;{ }^{* *} p<0.01$. (c) Representative result of immunofluorescence staining of E-cadherin (green) in the BCa cells after SIRT1-target-specific-siRNA treatment (KD) (B, D), compared with control-siRNA treatment (NC) (A, C). Nuclei (blue) were stained by DAPI. The scale bar for (D) is $40 \mu \mathrm{m}$. (d) Representative result of immunofluorescence staining of N-cadherin (green) in the BCa cells after SIRT1-target-specific-siRNA treatment (KD) (B, D), compared with control-siRNA treatment (NC) (A, C). Nuclei (blue) were stained by DAPI. The scale bars for (a) are $25 \mu \mathrm{m}$ and for (c-d) are $40 \mu \mathrm{m}$.

Indeed, in our SIRT1 deficiency cell model, we observed a significantly reduced ROS production (Figure 2). As we know, the role that ROS plays in cancer biology is quite complicated, which can be seen as a double-edged sword. A modest level of ROS is essential for tumorigenesis, whereas an excessive level would suppress tumors $[49,50]$. ROS can serve as a signal of either apoptosis or survival, which is determined by dosage, type, duration, and site of ROS production [51]. As Lin et al. reported, ROS produced in mitochondria could be the major source of ROS production and therefore lead to $\mathrm{BCa}$ cells apoptosis [52]. Therefore, the alteration of mitochondrial SOD2 was analyzed, which clears mitochondrial ROS, thus protecting cells from cell death [53]. Consistently, our result revealed an obvious upregulation of SOD2 protein (Figure 2), 

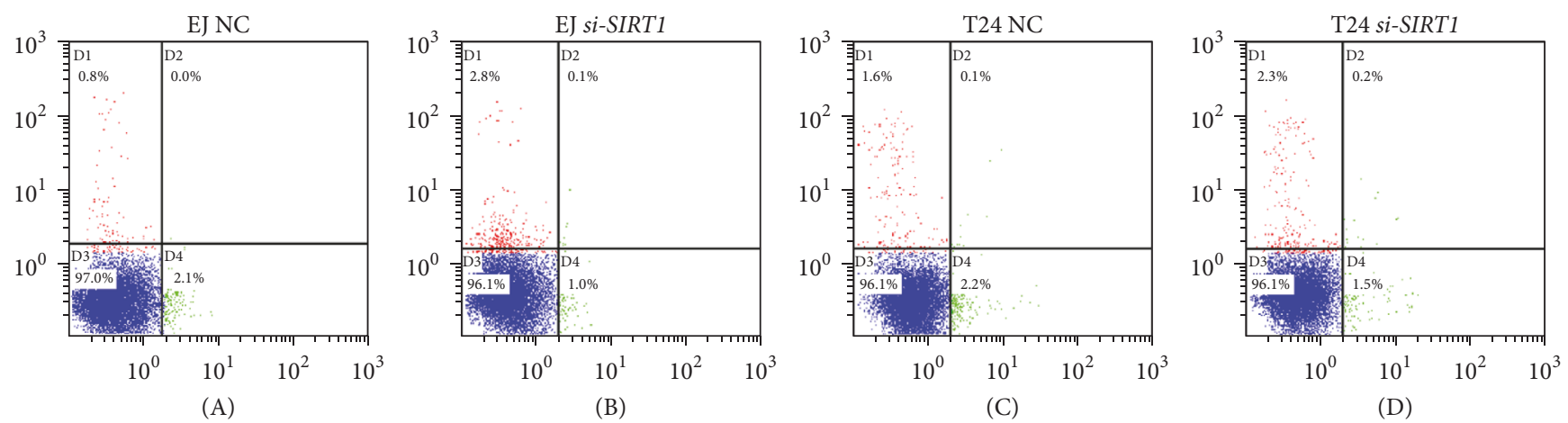

(a)

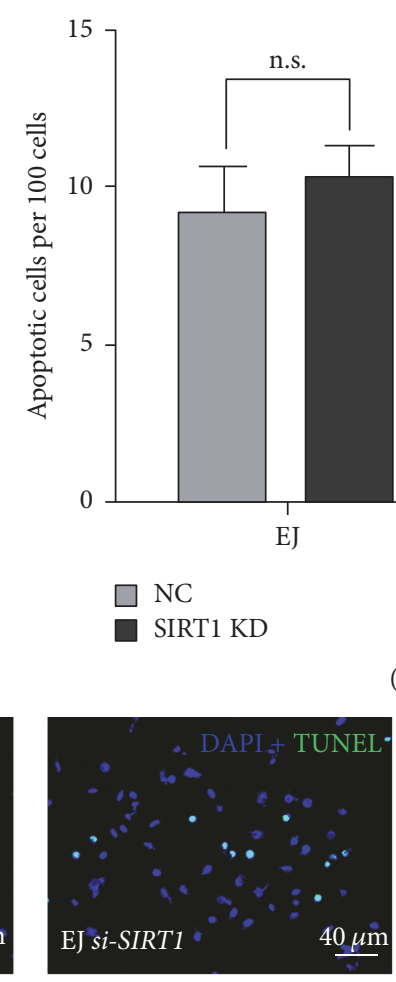

(B)

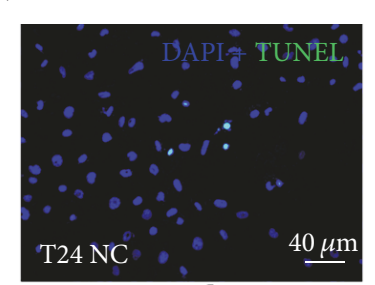

(C)

(c)

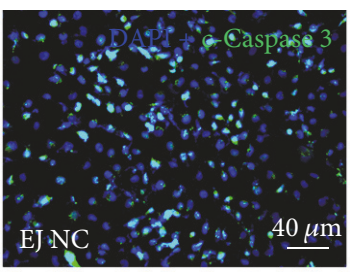

(A)

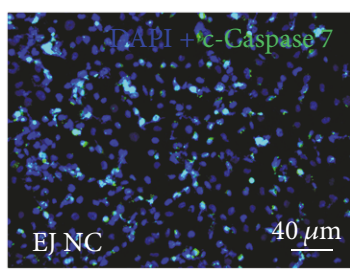

(A)

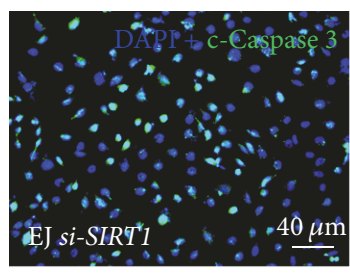

(B)

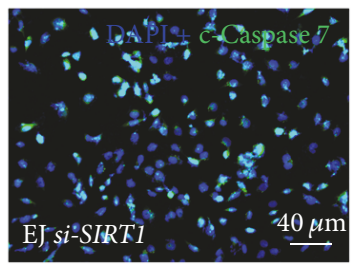

(B)

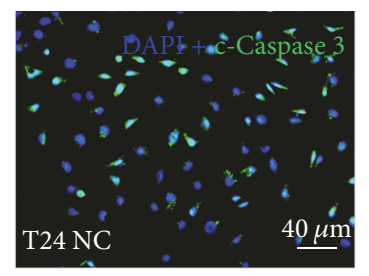

(C)

(d)

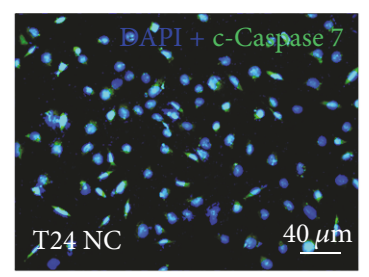

(C)

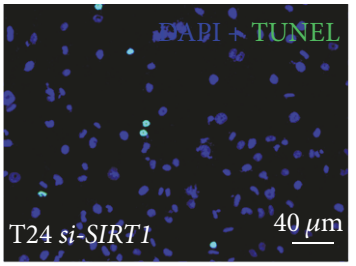

(D)

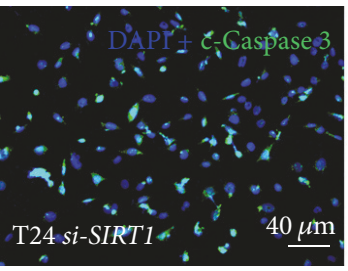

(D)

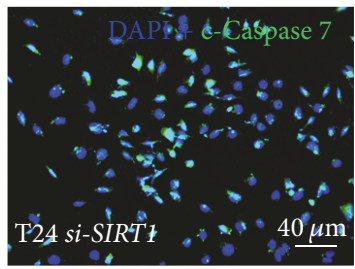

(D)

(e)

Figure 5: Continued. 


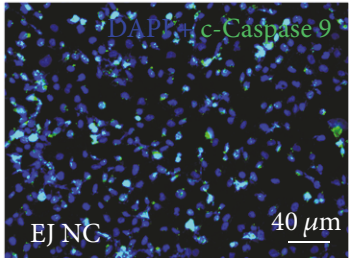

(A)

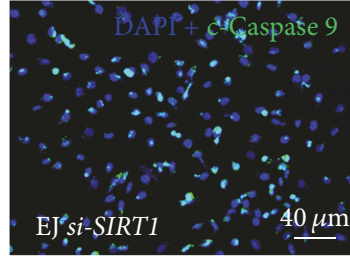

(B)

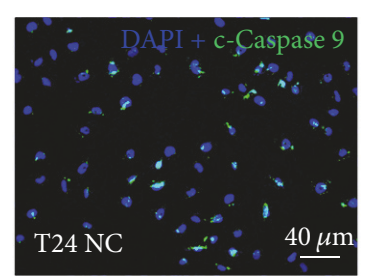

(C)

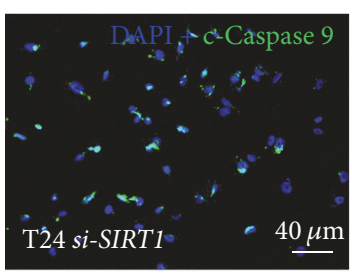

(D)

(f)

FIgURE 5: Decreased SIRT1 could not lead to BCa cell apoptosis. (a) The human bladder cancer cells EJ (contaminated by T24 as per "http://iclac.org/databases/cross-contaminations/") and T24 were transfected with SIRT1-target-specific-siRNA (B, D) and controlsiRNA (A, C) for $48 \mathrm{~h}$. BCa cells were stained with Annexin V/PI and apoptosis was measured by flow cytometry. (b) Statistical analysis of TUNEL-test revealed no significant (n.s.) increase of apoptotic cell rate in either EJ (contaminated by T24 as per "http://iclac.org/databases/cross-contaminations/") or T24 cells after SIRT1-target-specific-siRNA (SIRT1 KD) treatment. (c) TUNEL-test to detect apoptotic cells (green) in control-siRNA-treated BCa cells (A, C) and SIRT1-target-specific-siRNA-treated BCa cells (B, D). Nuclei (blue) were stained by DAPI. (d, e, f) Representative immunofluorescence images of cleaved-Caspases 3, 7, and 9 (green) in the T24 and EJ (contaminated by T24 as per "http://iclac.org/databases/cross-contaminations/") cells after SIRT1-target-specific-siRNA treatment (KD) (B, D), compared with control-siRNA treatment (NC) (A, C). Nuclei (blue) were stained by DAPI. The scale bars for (c-f) are $40 \mu \mathrm{m}$.

which may be responsible for no significant increase of $\mathrm{BCa}$ cells apoptosis observed (Figure 5). Another key enzyme involved in antioxidant response is Catalase, which has been reported to protect chromosomes against ionizing radiation [53] or oxidative damage [54]; therefore it suppresses cell death. And it also showed an increase in our study (Figure 2(f)), which may be another reason to explain that no significant increase of apoptosis occurred in the si-SIRT1 treated BCa cells (Figure 5).

Moreover, we observed a significantly induced cell cycle arrest at G0/G1 phase (Figure 3), followed by downregulation of proteins involved in G0/G1 to $S$ phase progression (CDK2/4/6). Cyclin-dependent kinases (CDKs) are a family of protein kinases playing vital roles in regulating cell cycle [55]. Among them CDK4 and CDK6 are responsible for helping cells getting out from G0 phase and into G1 phase $[56,57]$; meanwhile CDK2 plays its role in G1 phase [58]. Downregulation of CDK2/4/6 could lead to cell cycle arrest at G0/G1 phase [59], which may explain our result of flow cytometry analysis (Figure 2).

We highly suspect that the highly activated antioxidant response may play a vital role in the process avoiding the $\mathrm{BCa}$ cells with SIRT1 deficiency from apoptosis. However, further studies are needed for the detailed mechanism.

For further discussion, it is reported that the overexpression of Thromboxane-A 2 isoform- $\beta$ receptor $(T P \beta)$ plays an important role in the process of human bladder cancer, and TP agonist decreased acetylation of FOXO3 via upregulation of SIRT1 in the bladder cancer cell line UMUC3 [60]. However, no significant alteration of TP $\beta$ between human normal bladder and bladder cancer tissue was found in our microarray. It may suggest that there are other pathways regulating SIRT1 expression in bladder cancer. Moreover, as reported by Lin et al. [52], capsaicin inhibits tumor-associated NADH oxidase (tNOX) and SIRT1, thus changing multiple phenotypes of bladder cancer cells including apoptosis, cell cycle progression, and cell migration. In contrast, in our study we directly knocked down SIRT1, which could help us focus on the effect of SIRT1 downregulation, avoiding the interference of other factors.

\section{Conclusions}

Our study for the first time suggested that SIRT1 deficiency in bladder cancer cells could suppress proliferation and ROS production, as well as induce cell cycle arrest, possibly via the FOXO3a-mediated pathways.

\section{Disclosure}

All the funders had no role in study design, data collection and analysis, decision to publish, or preparation of the manuscript.

\section{Conflicts of Interest}

The authors declare that there are no conflicts of interest regarding the publication of this paper.

\section{Acknowledgments}

The excellent technical assistance of Yuan Zhu, Shanshan Zhang, and Danni Shan is gratefully acknowledged. The authors would like to thank Dr. Adam J. Hsu at Mayo Clinic, Rochester, USA, for critical reading and editing this manuscript. They also would like to acknowledge the KEGG database developed by Kanehisa Laboratories for the original source of KEGG pathway images "FOXO signaling pathway (map04068)." This study was supported in part by grants from the Natural Sciences Foundation of Hubei Province (Grant no. 42014CFA006), Medical Science and Technology Project of Zhejiang Province (Grant no. 2016KYB082), and National Natural Science Foundation of China (Grant no. 81300578). 


\section{References}

[1] M. Burger, J. W. F. Catto, G. Dalbagni et al., "Epidemiology and risk factors of urothelial bladder cancer," European Urology, vol. 63, no. 2, pp. 234-241, 2013.

[2] J. Ferlay, I. Soerjomataram, R. Dikshit et al., "Cancer incidence and mortality worldwide: sources, methods and major patterns in GLOBOCAN 2012," International Journal of Cancer, vol. 136, no. 5, pp. E359-E386, 2014.

[3] F.-C. Von Rundstedt, K. Rajapakshe, J. Ma et al., "Integrative Pathway Analysis of Metabolic Signature in Bladder Cancer: A Linkage to the Cancer Genome Atlas Project and Prediction of Survival," Journal of Urology, vol. 195, no. 6, pp. 1911-1919, 2016.

[4] F. Massari, C. Ciccarese, M. Santoni et al., "Metabolic phenotype of bladder cancer," Cancer Treatment Reviews, vol. 45, pp. 46-57, 2016.

[5] S.-P. Deng, L. Zhu, and D.-S. Huang, "Mining the bladder cancer-associated genes by an integrated strategy for the construction and analysis of differential co-expression networks," BMC Genomics, vol. 16, no. 3, article no. S4, 2015.

[6] R. A. Frye, "Characterization of five human cDNAs with homology to the yeast SIR2 gene: Sir2-like proteins (Sirtuins) metabolize NAD and may have protein ADP-ribosyltransferase activity," Biochemical and Biophysical Research Communications, vol. 260, no. 1, pp. 273-279, 1999.

[7] M. C. Haigis and D. A. Sinclair, "Mammalian sirtuins: biological insights and disease relevance," Annual Review of Pathology: Mechanisms of Disease, vol. 5, pp. 253-295, 2010.

[8] D. A. Sinclair and L. Guarente, "Extrachromosomal rDNA circles - A cause of aging in yeast," Cell, vol. 91, no. 7, pp. 10331042, 1997.

[9] M. Kaeberlein, M. McVey, and L. Guarente, "The SIR2/3/4 complex and SIR2 alone promote longevity in Saccharomyces cerevisiae by two different mechanisms," Genes \& Development, vol. 13, no. 19, pp. 2570-2580, 1999.

[10] R. A. Frye, "Phylogenetic classification of prokaryotic and eukaryotic Sir2-like proteins," Biochemical and Biophysical Research Communications, vol. 273, no. 2, pp. 793-798, 2000.

[11] N. Preyat and O. Leo, "Sirtuin deacylases: a molecular link between metabolism and immunity," Journal of Leukocyte Biology, vol. 93, no. 5, pp. 669-680, 2013.

[12] N.-K. Niu, Z.-L. Wang, S.-T. Pan et al., "Pro-apoptotic and proautophagic effects of the aurora kinase A inhibitor alisertib (MLN8237) on human osteosarcoma U-2 OS and MG-63 cells through the activation of mitochondria-mediated pathway and inhibition of p38 MAPK/PI3K/Akt/mTOR signaling pathway," Drug Design, Development and Therapy, vol. 9, pp. 1555-1584, 2015.

[13] Z.-W. Zhou, X.-X. Li, Z.-X. He et al., "Induction of apoptosis and autophagy via sirtuinl- and PI3K/Akt/mTOR-mediated pathways by plumbagin in human prostate cancer cells," Drug Design, Development \& Therapy, vol. 9, pp. 1511-1554, 2015.

[14] F. D. Heyward, D. Gilliam, M. A. Coleman et al., "Obesity weighs down memory through a mechanism involving the neuroepigenetic dysregulation of Sirt1," Journal of Neuroscience, vol. 36, no. 4, pp. 1324-1335, 2016.

[15] F. Xu, X. Zheng, B. Lin et al., "Diet-induced obesity and insulin resistance are associated with brown fat degeneration in SIRT1deficient mice," Obesity, vol. 24, no. 3, pp. 634-642, 2016.

[16] S. Mariani, D. Costantini, C. Lubrano et al., "Circulating SIRT1 inversely correlates with epicardial fat thickness in patients with obesity," Nutrition, Metabolism and Cardiovascular Diseases, vol. 26, no. 11, pp. 1033-1038, 2016.

[17] J. Han, M. Wei, Q. Wang et al., "Association of genetic variants of SIRT1 with Type 2 diabetes mellitus," Gene Expression, vol. 16, no. 4, pp. 177-185, 2015.

[18] S. Venkatasubramanian, R. M. Noh, S. Daga et al., "Effects of the small molecule SIRT1 activator, SRT2104 on arterial stiffness in otherwise healthy cigarette smokers and subjects with type 2 diabetes mellitus," Open Heart, vol. 3, no. 1, p. e000402, 2016.

[19] J. Xu, N. Li, J. Wang, C. Zhang, S. Ding, Y. Jiao et al., "Effect of metformin on the expression of SIRT1 and UCP2 in rat liver of type 2 diabetes mellitus and nonalcoholic fatty liver," Zhong nan da xue xue bao Yi xue ban, vol. 38, no. 9, pp. 882-827, 2013.

[20] Y. Colak, O. Ozturk, E. Senates et al., "SIRT1 as a potential therapeutic target for treatment of nonalcoholic fatty liver disease," Medical Science Monitor, vol. 17, no. 5, article HY5-9, 2011.

[21] T. Ren, C. Huang, and M. Cheng, "Dietary blueberry and bifidobacteria attenuate nonalcoholic fatty liver disease in rats by affecting SIRT1-mediated signaling pathway," Oxidative Medicine and Cellular Longevity, vol. 2014, Article ID 469059, 2014.

[22] Y. R. Chung, H. Kim, S. Y. Park et al., "Distinctive role of SIRT1 expression on tumor invasion and metastasis in breast cancer by molecular subtype," Human Pathology, vol. 46, no. 7, pp. 1027$1035,2015$.

[23] M. F. Santolla, S. Avino, M. Pellegrino et al., "SIRT1 is involved in oncogenic signaling mediated by GPER in breast cancer," Cell Death and Disease, vol. 6, no. 7, Article ID e1834, 2015.

[24] S. M. Rizk, N. N. Shahin, and O. G. Shaker, "Association between SIRT1 gene polymorphisms and breast cancer in Egyptians," PLoS ONE, vol. 11, no. 3, Article ID e0151901, 2016.

[25] T. Shuang, M. Wang, Y. Zhou, and C. Shi, "Over-expression of Sirtl contributes to chemoresistance and indicates poor prognosis in serous epithelial ovarian cancer (EOC)," Medical Oncology, vol. 32, no. 12, article no. 260, 2015.

[26] L. Sun, H. Li, J. Chen et al., "PIASy mediates hypoxiainduced SIRT1 transcriptional repression and epithelial-tomesenchymal transition in ovarian cancer cells," Journal of Cell Science, vol. 126, no. 17, pp. 3939-3947, 2013.

[27] D. Li, F.-F. Bi, N.-N. Chen et al., "A novel crosstalk between BRCA1 and sirtuin 1 in ovarian cancer," Scientific Reports, vol. 4, article no. 6666, 2014.

[28] S. Singh, P. U. Kumar, S. Thakur et al., "Expression/localization patterns of sirtuins (SIRT1, SIRT2, and SIRT7) during progression of cervical cancer and effects of sirtuin inhibitors on growth of cervical cancer cells," Tumor Biology, vol. 36, no. 8, pp. 61596171, 2015.

[29] S. J. Allison, M. Jiang, and J. Milner, "Oncogenic viral protein HPV E7 up-regulates the SIRT1 longevity protein in human cervical cancer cells.", Aging, vol. 1, no. 3, pp. 316-327, 2009.

[30] M. Teramae, T. Fukuda, T. Wada, M. Kawanishi, K. Imai, M. Yamauchi et al., "Sirtuinl expression predicts the efficacy of neoadjuvant chemotherapy for locally advanced uterine cervical cancer," Mol Clin Oncol, vol. 3, no. 1, pp. 73-78, 2015.

[31] J. Li, G. Dong, B. Wang, W. Gao, and Q. Yang, "MiR-543 promotes gastric cancer cell proliferation by targeting SIRT1," Biochemical and Biophysical Research Communications, vol. 469, no. 1, pp. 15-21, 2016.

[32] A. Noguchi, K. Kikuchi, H. C. Zheng et al., "SIRT1 expression is associated with a poor prognosis, whereas $\mathrm{DBCl}$ is associated 
with favorable outcomes in gastric cancer," Cancer Medicine, vol. 3, no. 6, pp. 1553-1561, 2014.

[33] L. Zhang, X. Wang, and P. Chen, "MiR-204 down regulates SIRT1 and reverts SIRT1-induced epithelial-mesenchymal transition, anoikis resistance and invasion in gastric cancer cells," BMC Cancer, vol. 13, article 290, 2013.

[34] J. D. Lovaas, L. Zhu, C. Y. Chiao, V. Byles, D. V. Faller, and Y. Dai, "SIRT1 enhances matrix metalloproteinase-2 expression and tumor cell invasion in prostate cancer cells," Prostate, vol. 73, no. 5, pp. 522-530, 2013.

[35] D. Wu, S. Yu, L. Jia et al., "Orphan nuclear receptor TLX functions as a potent suppressor of oncogene-induced senescence in prostate cancer via its transcriptional co-regulation of the CDKN1A (p21WAF1/CIP1) and SIRT1 genes," Journal of Pathology, vol. 236, no. 1, pp. 103-115, 2015.

[36] V. Byles, L. Zhu, J. D. Lovaas et al., "SIRT1 induces EMT by cooperating with EMT transcription factors and enhances prostate cancer cell migration and metastasis," Oncogene, vol. 31, no. 43, pp. 4619-4629, 2012.

[37] R. Cao, Z. Meng, T. Liu et al., "Decreased TRPM7 inhibits activities and induces apoptosis of bladder cancer cells via ERK1/2 pathway," Oncotarget, vol. 7, no. 45, pp. 72941-72960, 2016.

[38] G. Wang, R. Cao, Y. Wang et al., "Simvastatin induces cell cycle arrest and inhibits proliferation of bladder cancer cells via PPAR $\gamma$ signalling pathway," Scientific Reports, vol. 6, Article ID 35783, 2016.

[39] K. Qian, G. Wang, R. Cao et al., “Capsaicin Suppresses cell proliferation, induces cell cycle arrest and ros production in bladder cancer cells through FOXO3a-mediated pathways," Molecules (Basel, Switzerland), vol. 21, no. 10, 2016.

[40] W. Yu, Y. Zhang, L. Xu, S. Sun, X. Jiang, and F. Zhang, "Microarray-based bioinformatics analysis of osteoblasts on TiO2 nanotube layers," Colloids and Surfaces B: Biointerfaces, vol. 93, pp. 135-142, 2012.

[41] Dennis G Jr., B. T. Sherman, D. A. Hosack, J. Yang, W. Gao, and H. C. Lane, "Database for annotation, visualization, and integrated discovery," Genome Biol, vol. 4, no. 5, 2003.

[42] M. Kanehisa, Y. Sato, M. Kawashima, M. Furumichi, and M. Tanabe, "KEGG as a reference resource for gene and protein annotation," Nucleic Acids Research, vol. 44, no. 1, pp. D457D462, 2016.

[43] M. Kanehisa and S. Goto, "KEGG: kyoto encyclopedia of genes and genomes," Nucleic Acids Research, vol. 28, no. 1, pp. 27-30, 2000.

[44] R. Frazzi, R. Valli, I. Tamagnini, B. Casali, N. Latruffe, and F. Merli, "Resveratrol-mediated apoptosis of hodgkin lymphoma cells involves SIRT1 inhibition and FOXO3a hyperacetylation," International Journal of Cancer, vol. 132, no. 5, pp. 1013-1021, 2013.

[45] T. Scholzen and J. Gerdes, “The Ki-67 protein: from the known and the unknown," Journal of Cellular Physiology, vol. 182, no. 3, pp. 311-322, 2000.

[46] D. L. McClelland Descalzo, T. S. Satoorian, L. M. Walker, N. R. L. Sparks, P. Y. Pulyanina, and N. I. zur Nieden, "Glucose-Induced Oxidative Stress Reduces Proliferation in Embryonic Stem Cells via FOXO3A/ $\beta$-Catenin-Dependent Transcription of p21cip1," Stem Cell Reports, vol. 7, no. 1, pp. 55-68, 2016.

[47] B. Zeng, Y. Li, B. Niu et al., "Involvement of PI3K/Akt/FoxO3a and PKA/CREB Signaling Pathways in the Protective Effect of Fluoxetine Against Corticosterone-Induced Cytotoxicity in
PC12 Cells," Journal of Molecular Neuroscience, vol. 59, no. 4, pp. 567-578, 2016.

[48] S. Shibata, H. Ishitobi, S. Miyaki, T. Kawaoka, T. Kayashima, and K. Matsubara, "Carnosic acid protects starvation-induced SHSY5Y cell death through Erk1/2 and Akt pathways, autophagy, and FoxO3a," International Journal of Food Sciences and Nutrition, vol. 67, no. 8, pp. 977-982, 2016.

[49] D. Trachootham, Y. Zhou, H. Zhang et al., "Selective killing of oncogenically transformed cells through a ROS-mediated mechanism by beta-phenylethyl isothiocyanate," Cancer Cell, vol. 10, no. 3, pp. 241-252, 2006.

[50] B. Halliwell, "Oxidative stress and cancer: have we moved forward?” Biochemical Journal, vol. 401, no. 1, pp. 1-11, 2007.

[51] S. C. Gupta, D. Hevia, S. Patchva, B. Park, W. Koh, and B. B. Aggarwal, "Upsides and downsides of reactive oxygen species for Cancer: the roles of reactive oxygen species in tumorigenesis, prevention, and therapy," Antioxidants and Redox Signaling, vol. 16, no. 11, pp. 1295-1322, 2012.

[52] M.-H. Lin, Y.-H. Lee, H.-L. Cheng, H.-Y. Chen, F.-H. Jhuang, and P. J. Chueh, "Capsaicin inhibits multiple bladder cancer cell phenotypes by inhibiting tumor-associated NADH oxidase (tNOX) and sirtuin1 (SIRT1)," Molecules, vol. 21, no. 7, article no. 849, 2016.

[53] X. Xiao, H. Luo, K. N. Vanek, A. C. LaRue, B. A. Schulte, and G. Y. Wang, "Catalase inhibits ionizing radiation-induced apoptosis in hematopoietic stem and progenitor cells," Stem Cells and Development, vol. 24, no. 11, pp. 1342-1351, 2015.

[54] Y. S. Park, S. Y. You, S. Cho et al., "Eccentric localization of catalase to protect chromosomes from oxidative damages during meiotic maturation in mouse oocytes," Histochemistry and Cell Biology, vol. 146, no. 3, pp. 281-288, 2016.

[55] M. Malumbres, "Cyclin-dependent kinases," Genome Biology, vol. 15, no. 6, p. 122, 2014.

[56] Y.-X. Zhang, E. Sicinska, J. T. Czaplinski et al., "Antiproliferative effects of CDK4/6 inhibition in CDK4-amplified human liposarcoma in vitro and in vivo," Molecular Cancer Therapeutics, vol. 13, no. 9, pp. 2184-2193, 2014.

[57] M. Zanuy, A. Ramos-Montoya, O. Villacañas et al., "Cyclindependent kinases 4 and 6 control tumor progression and direct glucose oxidation in the pentose cycle," Metabolomics, vol. 8, no. 3, pp. 454-464, 2012.

[58] T. A. Chohan, H. Qian, Y. Pan, and J.-Z. Chen, "Cyclindependent kinase-2 as a target for cancer therapy: Progress in the development of CDK2 inhibitors as anti-cancer agents," Current Medicinal Chemistry, vol. 22, no. 2, pp. 237-263, 2015.

[59] J. K. Buolamwini, "Cell cycle molecular targets in novel anticancer drug discovery," Current Pharmaceutical Design, vol. 6, no. 4, pp. 379-392, 2000.

[60] P. M. Sobolesky, P. V. Halushka, E. Garrett-Mayer, M. T. Smith, and O. Moussa, "Regulation of the Tumor Suppressor FOXO3 by the Thromboxane-A2 Receptors in urothelial cancer," PLoS ONE, vol. 9, no. 9, Article ID e0107530, 2014. 


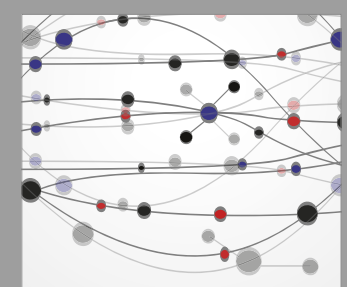

The Scientific World Journal
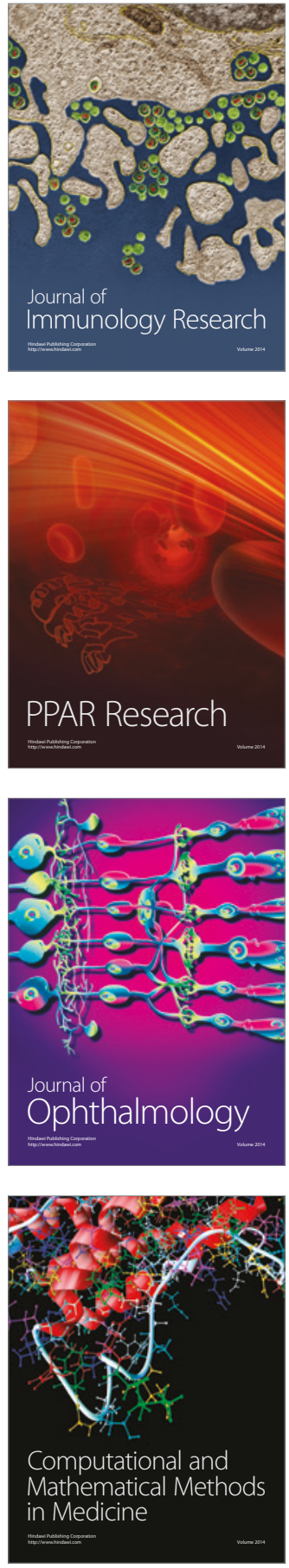

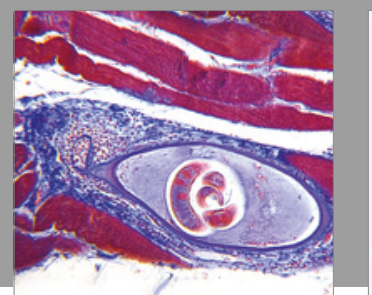

Gastroenterology Research and Practice
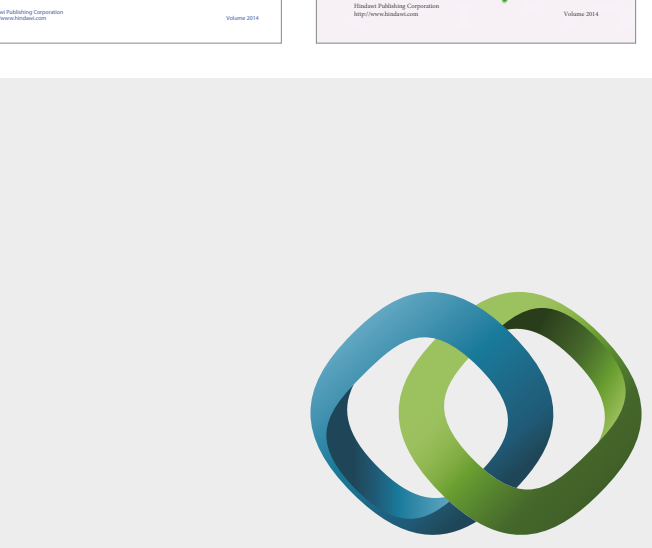

\section{Hindawi}

Submit your manuscripts at

https://www.hindawi.com
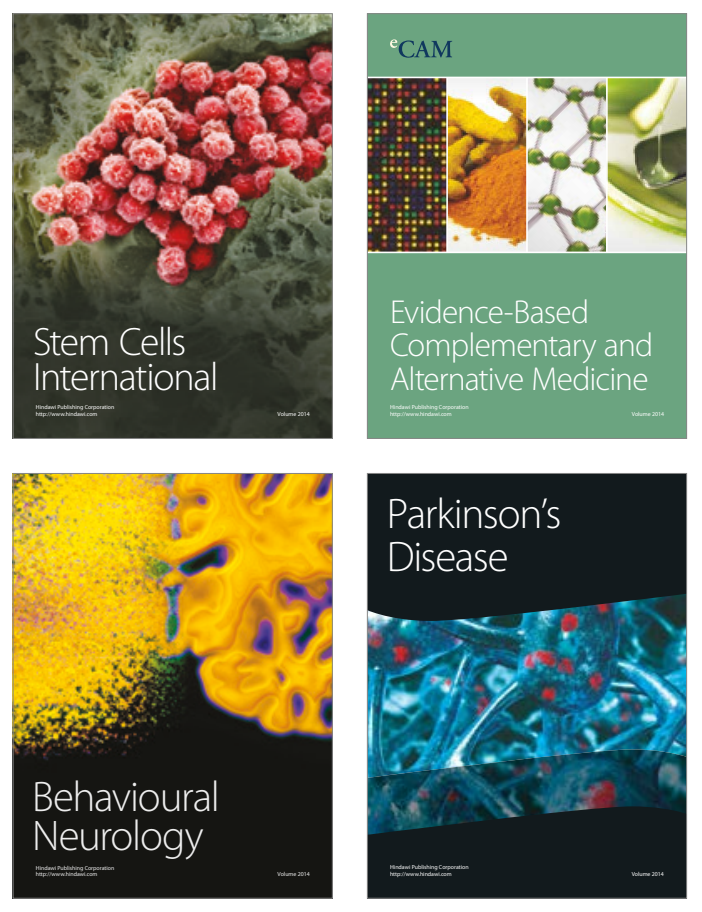
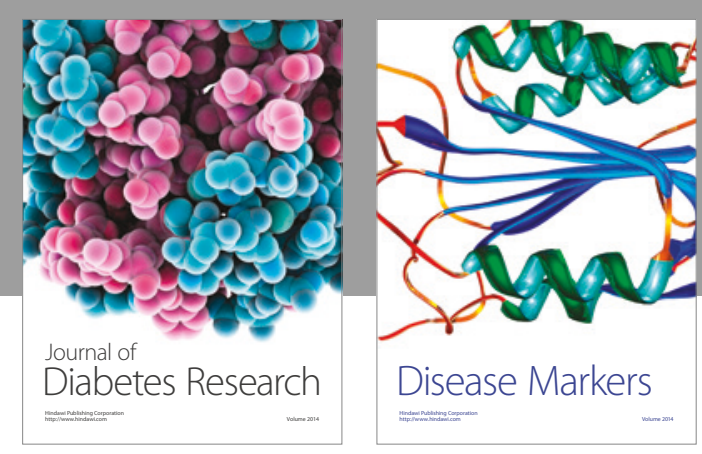

Disease Markers
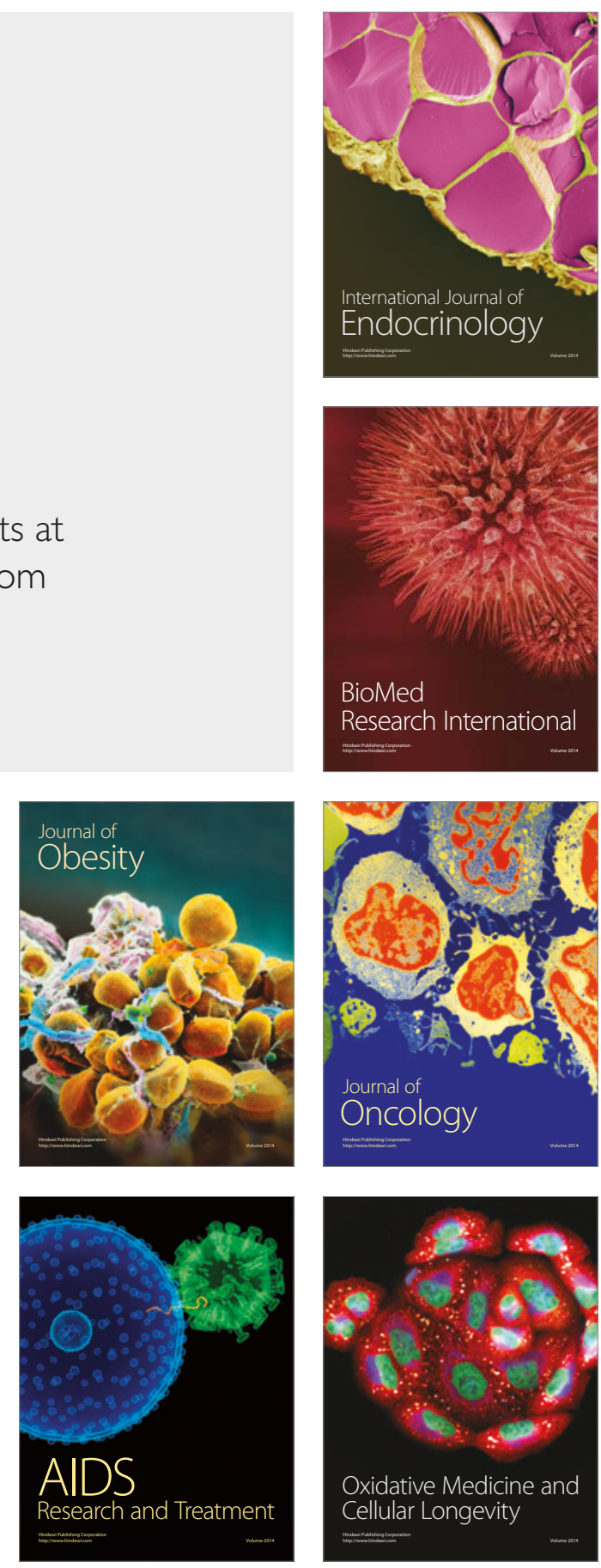